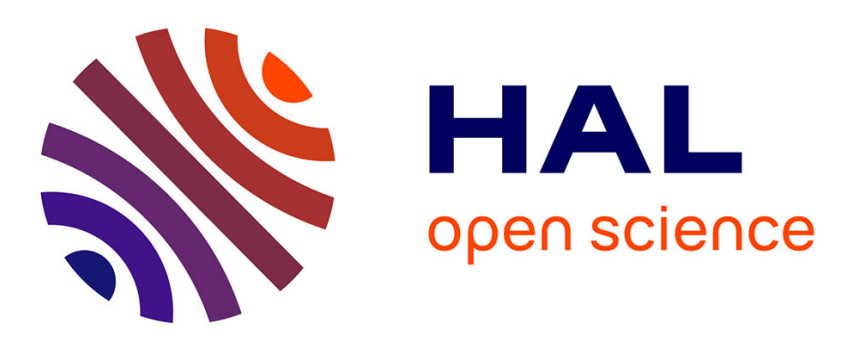

\title{
A coherent population trapping Cs vapor cell atomic clock based on push-pull optical pumping
}

Moustafa Abdel Hafiz, Rodolphe Boudot

\section{To cite this version:}

Moustafa Abdel Hafiz, Rodolphe Boudot. A coherent population trapping Cs vapor cell atomic clock based on push-pull optical pumping. Journal of Applied Physics, 2015, 118 (12), pp.124903. hal02868415

\section{HAL Id: hal-02868415 \\ https://hal.science/hal-02868415}

Submitted on 15 Jun 2020

HAL is a multi-disciplinary open access archive for the deposit and dissemination of scientific research documents, whether they are published or not. The documents may come from teaching and research institutions in France or abroad, or from public or private research centers.
L'archive ouverte pluridisciplinaire HAL, est destinée au dépôt et à la diffusion de documents scientifiques de niveau recherche, publiés ou non, émanant des établissements d'enseignement et de recherche français ou étrangers, des laboratoires publics ou privés. 


\title{
A coherent population trapping Cs vapor cell atomic clock based on push-pull optical pumping
}

\author{
Moustafa Abdel Hafiz and Rodolphe Boudot \\ FEMTO-ST, CNRS, UFC, 26 chemin de l'Epitaphe 25030 Besançon Cedex, France
}

(Dated: September 28, 2015)

This article reports on the characterization of a compact coherent-population-trapping (CPT) Cs vapor cell atomic clock using the push-pull optical pumping technique (PPOP). This method allows to detect high-contrast CPT resonances on the 0-0 magnetic-field insensitive clock transition. The clock short-term fractional frequency stability is measured to be $3 \times 10^{-13} \tau^{-1 / 2}$ up to 100 seconds averaging time, in correct agreement with the signal-to-noise ratio limit. These results are among the best performances ever reported for a CPT-based clock. The noise budget is detailed. A preliminar study is reported to evaluate limitations to the clock mid-term frequency stability. The clock performances are found to be presently mainly limited in the mid-term by laser power effects.

PACS numbers: 06.30.Ft, 32.70.Jz, 32.70.Jz 


\section{INTRODUCTION}

Microwave vapor cell atomic clocks, using basically interactions between optically-pumped alkali atoms in a vapor cell and resonant laser light, are well-adapted to be exploited as secondary frequency standards in numerous applications where quartz crystal oscillators can not reach frequency stability requirements and where simultaneously size, power consumption and cost are of relevant importance. In that sense, lamp-pumped $\mathrm{Rb}$ atomic clocks, exhibiting fractional frequency stability in the $0.5-1 \times 10^{-11}$ range at $1 \mathrm{~s}$ integration time, are implemented widely in satellite-based navigation systems [1-3], telecommunications or defense and laboratory equipments. Over the last years, thanks to the use of better performing laser sources and dedicated techniques, laboratory-prototype vapor cell atomic clocks, based on optical-microwave double resonance technique $[4,5]$ or coherent population trapping (CPT) [6], have demonstrated short-term fractional frequency stability in the $1-4 \times 10^{-13} \tau^{-1 / 2}$ range. These performances are about two orders of magnitude better than commercially-available $\mathrm{Rb}$ clocks, making them even competitive with passive hydrogen masers. Simultaneously, research activities are intensively pursued in numerous laboratories and industries to explore and push the limits of this technology whose ultimate frequency stability performances are predicted to be at the level of $1-2 \times 10^{-14}$ at $1 \mathrm{~s}$ averaging time. This underlies that a large marge of progress remains to be accomplished and demonstrated with gas cell standards.

In this domain, INRIM has developed a pulsed optically pumped (POP) Rb frequency standard with microwave [7] and optical detection [8] that exhibits a fractional frequency stability of $1.2 \times 10^{-12} \tau^{-1 / 2}$ and $1.7 \times 10^{-13} \tau^{-1 / 2}$ up to $10000 \mathrm{~s}$ integration time respectively. Recently, a chinese group inspired by the work of Zhu et al. [9] proposed a polarization-selective method for the signal detection in such systems. This technique allowed to demonstrate resonance contrasts up to $90 \%$ and a frequency stability of $2 \times 10^{-12} \tau^{-1 / 2}$ up to $1000 \mathrm{~s}$ averaging times [10]. LTF-UNINE has demonstrated a high-performance optically pumped Rb clock based on a compact magnetrontype microwave cavity using continuous [11] or pulsed interaction [12] with a fractional frequency stability of $2.4 \times 10^{-13} \tau^{-1 / 2}$ and $2.1 \times 10^{-13} \tau^{-1 / 2}$ up to 100 s averaging time respectively.

An alternative to double-resonance $\mathrm{Rb}$ clocks for the development of high-performance vapor cell clocks is the use of coherent population trapping physics $[13,14]$. INRIM has developed a CPT maser with a short term fractional frequency stability of $3 \times 10^{-12} \tau^{-1 / 2}$, value close to the theoretical prediction [15]. LNE-SYRTE has developed an original CPT clock where the detection of high-contrast and narrow Raman-Ramsey fringes is allowed thanks to the combination of a specific lin $\perp$ lin interaction scheme and a pulsed interrogation [16]. The Ramsey interrogation [17] allows to 
reduce greatly light shift phenomena [18] and to avoid the CPT resonance power broadening [19]. Recently, this pulsed Cs CPT clock has demonstrated a short term fractional frequency stability of $3.2 \times 10^{-13} \tau^{-1 / 2}$ up to averaging times of $1000 \mathrm{~s}$ [20]. A drawback of this table-top prototype clock is the use two phase-locked lasers that make the system voluminous and complex. Moreover, the Dick effect or intermodulation effect [21, 22] contribution is increased due to microwave phase noise degradation through the optical phase lock loop (OPLL) [20]. Different CPT clock systems [23-26] with optimized CPT pumping schemes [27-30] and single modulated laser sources were proposed in the literature. Nevertheless, no clock frequency stability results were reported in these articles.

In the frame of the MClocks project [31], we recently started in FEMTO-ST the development of a compact Cs CPT atomic clock based on the push-pull optical pumping (PPOP) technique, pioneerly proposed by Jau et al. [27]. This method consists to make the atoms interact with a bichromatic optical field that alternates between right and left circular polarization at the clock Bohr frequency and was recently used to develop a high-sensitivity optical magnetometer [32]. In recent literature, using this setup, we reported the spectroscopy of high-contrast CPT resonances in Cs vapor cells [33] and demonstrated the possibility to detect high-contrast Raman-Ramsey fringes [34]. Including and highlighting different modifications to the experimental setup, this article aims to report first short-term frequency-stability results of our CPT-PPOP based Cs vapor cell atomic clock. A promising short-term fractional frequency stability of $3 \times 10^{-13} \tau^{-1 / 2}$ up to $\tau=100 \mathrm{~s}$ integration time in $\mathrm{CW}$ regime, in correct agreement with the noise budget, is demonstrated.

\section{EXPERIMENTAL SET-UP}

Figure 1 presents the Cs CPT clock experimental set-up. The laser source is a commerciallyavailable GaAs semi-conductor $1 \mathrm{MHz}$-linewidth distributed-feedback (DFB) diode laser tuned at $894 \mathrm{~nm}$ on the Cs $\mathrm{D}_{1}$ line [35]. A Faraday optical isolator (not shown on Fig. 1) is placed at the output of the DFB laser in order to avoid optical feedback. A MZ EOM (Photline NIRMX800-LN-10) driven at $4.596 \mathrm{GHz}$ with a low noise microwave frequency synthesizer inspired by [36] is used to generate phase-coherent optical sidebands frequency-separated by $9.192 \mathrm{GHz}$. The local oscillator consists of a high-spectral purity $100 \mathrm{MHz}$ quartz-crystal oscillator phase-locked to a high-stability $10 \mathrm{MHz}$ quartz source and then frequency-multiplied to $4.596 \mathrm{GHz}$. At the output of the EOM, optical carrier rejection is actively stabilized thanks to an original microwave synchronous detector presented in [33]. For this purpose, a fraction of the laser power is detected 
at the output of the EOM with a fast photodiode (FPD). The demodulated $4.6 \mathrm{GHz}$ signal is compared to the $4.6 \mathrm{GHz}$ local oscillator (LO) signal using a microwave double-balanced mixer. The latter delivers an error signal, processed through a proportional-integral (PI) controller and used to correct the EOM dc bias voltage value. The carrier rejection is ultimately limited by the EOM extinction ratio measured to be $24 \mathrm{~dB}$ with our device. Figure 2 reports the EOM power transmission versus the EOM temperature. A turnover point at a temperature of about $40.2^{\circ} \mathrm{C}$ is obtained where the optical power transmission is maximized and where the EOM transmission sensitivity to temperature variations is canceled at the first order. The fiber modulator ensemble is inserted into a box with insulating foam and is actively temperature-stabilized to the $\mathrm{mK}$ level at $40.2^{\circ} \mathrm{C}$.

A Cs reference cell is used to stabilize the laser frequency. Our experiment is similar to a saturated absorption scheme setup. The main difference is that, in our case, a dual-frequency laser system is used, inducing that two different transitions are involved in the saturated absorption scheme $\left(F=3 \rightarrow F^{\prime}=4\right.$ and $\left.F=4 \rightarrow F^{\prime}=4\right)$. For this purpose, the laser beam at the output of the EOM, that carries both optical lines frequency-split by $9.192 \mathrm{GHz}$, is sent into an annex reference Cs cell, reflected back by a mirror and detected by a photodiode. A quarter-wave plate is inserted between the cell and the reflection mirror, so that the incoming and reflected beams are linearly and orthogonally polarized. The cell is surrounded by a single-layer mu-metal magnetic shield. No static magnetic field is applied. The cell is not temperature-controlled. Figure 3 (curve a) plots the optical absorption lines detected in the reference evacuated cell. Under given polarization conditions described above, an original shape of the absorption line with a large increase in Dopplerfree atomic absorption is observed. We checked that the logarithm of the data in Fig. 3 (curve a) is well fitted by an absorption doublet, each absorption profile being composed by the sum of a broad Doppler-induced Gaussian profile and a narrow lorentzian profile. The narrow dip linewidth is about $15 \mathrm{MHz}$. The Doppler width is about $380 \mathrm{MHz}$, in good agreement with theoretical expectations [39]. We observed that the narrow absorption dip could be reversed by rotating the quarter-wave plate or introducing a static magnetic field. We believe that this behavior is somewhat analog to the one described in [40]. In this article, the possibility to reverse peaks in cesium saturation spectroscopy (enhanced transmission to enhanced absorption) was demonstrated and explained, under various polarization configurations, with and without applied magnetic fields, on $\mathrm{Cs}$ atom $\mathrm{D}_{2}$ line. In our experiment, an analog system is studied on the Cs $\mathrm{D}_{1}$ line, the only difference being the fact that a dual-frequency laser system is used. Further experimental and theoretical studies are under progress. The laser is frequency-stabilized by modulating the DFB 
laser current at $62 \mathrm{kHz}$ and demodulating it with a lock-in amplifier LA1. The laser frequency servo bandwidth is about $1.5 \mathrm{kHz}$.

Figure 3 (curve b) plots the optical absorption line detected in the buffer-gas filled CPT cell. The latter consists of a 2-cm diameter and 5-cm long cylindrical Cs vapor cell filled with a $\mathrm{N}_{2}$-Ar buffer gas mixture of total pressure 15.3 Torr and partial pressure ratio $r=P_{A r} / P_{N_{2}}=0.4$ to operate in the Dicke regime [37]. Some basic tests were also performed using a 2-cm long and 2-cm diameter cell filled with 21 Torr of $\mathrm{N}_{2}$-Ar mixture $(r=0.6)$. The linewidth of the optical absorption line in the CPT cell is measured to be $594 \mathrm{MHz}$, in correct agreement with a theoretical value of 617 $\mathrm{MHz}$ [38]. A frequency shift of $-122 \mathrm{MHz}$ is measured between the pure Cs cell and the CPT cell, also in good agreement with [38].

At the output of the EOM, an AOM-based laser power stabilization system with a servo bandwidth of about $20 \mathrm{kHz}$ is implemented. In this servo loop, a fraction of the first-order (order -1 ) output beam is detected by the photodiode PD1. The resulting voltage signal is compared to an ultra-lowdrift reference voltage (LM399) to produce an error signal and to correct the microwave power of the synthesizer that drives the AOM at $122 \mathrm{MHz}$. The order -1 at the output of the AOM is sent to the CPT cell to shift the laser frequency by $-122 \mathrm{MHz}$ and to compensate for the buffer-gas induced optical frequency shift.

A Michelson delay-line and polarization orthogonalizer system, based on two arms each composed of a mirror and a quarter-wave plate, is used to generate two time-delayed orthogonally polarized optical fields. An optical path length difference $x$, multiple of the quarter of the Cs atom clock transition wavelength $\lambda_{c s}\left(x=n \lambda_{c s} / 4=n \times 8.1 \mathrm{~mm}\right.$ with $n=1,3,5 .$. an odd integer $)$, is adjusted between both arms of the system to maximize the number of atoms in the 0-0 clock transition [27]. A last quarter wave plate allows to produce a bichromatic optical field that alternates between right and left circular polarization in order to realize the PPOP interaction scheme. At the output of the Michelson system, the diameter beam is expanded to $2 \mathrm{~cm}$ thanks to a pair of convergent optical lenses to cover the whole diameter of the vapor cell atomic resonator.

For most of the experiments, the cell temperature $T_{\text {cell }}$ is stabilized at about $35^{\circ} \mathrm{C}$ where the CPT signal height is maximized. This optimum results from a trade-off between the increase of atomic density and the increase of the vapor optical thickness [41, 42]. Typical cell fluctuations, as reported later, are well below the $\mathrm{mK}$ level for integration times up to $10000 \mathrm{~s}$ averaging time. The presence of buffer gas in the CPT cell causes a collisional frequency shift of the Cs clock transition of about $9.3 \mathrm{kHz}$. We note the clock frequency $\nu_{c} \sim \nu_{0}+9.3 \mathrm{kHz}$ where $\nu_{0}$ is the unperturbed Cs atom clock frequency $(9.192631770 \mathrm{GHz})$. For all measurements of the article (except for figure 
4), a static magnetic field of $89 \mathrm{mG}$ parallel to the laser beam propagation direction is applied to split the hyperfine ground-state Zeeman transitions. The ensemble is surrounded by a doublelayer mu-metal magnetic shield. The CPT resonance is monitored by detecting the laser power transmitted through the cell using the low-noise photodiode PD2. The resulting signal is analyzed by a computer that drives the local oscillator frequency. The atomic clock closed-loop operation is achieved by a conventional synchronous modulation-demodulation technique at a frequency $f_{m}$ of $125 \mathrm{~Hz}$. For frequency stability measurements, the clock output signal is compared to the signal of a state-of-the-art reference active hydrogen maser from T4Science [43]. The fractional frequency stability of the hydrogen maser, measured against a cryogenic sapphire oscillator [44], is $8 \times 10^{-14}$ and $3 \times 10^{-15}$ at $\tau=1 \mathrm{~s}$ and $100 \mathrm{~s}$ respectively. These performances are below those of the CPT clock and do not limit the measurement.

\section{CLOCK SIGNAL AND NOISE SOURCES}

\section{A. Clock short-term frequency stability}

Let remind first the impact of the push-pull interaction scheme onto the clock signal. Figure 4 shows a typical Zeeman spectrum detected in a 2-cm long and 2-cm diameter buffer-gas filled cell in respective cases where standard circular polarization $\left(\sigma^{+}\right)$or PPOP scheme is used. The total laser power incident in the cell is $400 \mu \mathrm{W}$. For this figure, the static magnetic field is reduced to $24.5 \mathrm{mG}$ and the cell temperature is $38^{\circ} \mathrm{C}$. With circular polarization, it is shown that most of the atoms are transferred in extreme Zeeman sub-levels and do not participate to the clock transition. With the PPOP scheme, the 0-0 clock transition is greatly favored and the Zeeman spectrum gets symmetrical. In this example, the contrast $C$ of the 0-0 clock transition, defined as $C=S / y_{0}$ where $S$ is the CPT signal height and $y_{0}$ is the dc background level, is $11.5 \%$ whereas it is only $1.4 \%$ with circular polarization.

The short-term fractional frequency stability $\sigma_{y}(\tau)$ of a passive atomic clock is well-approximated by $[45]$ :

$$
\sigma_{y}(\tau) \sim \frac{\Delta \nu}{\nu_{c}} \frac{1}{S N R} \tau^{-1 / 2}
$$

where $\Delta \nu$ is the clock resonance full-width at half maximum (FWHM), $\nu_{c}$ is the clock transition frequency, $S N R$ is the signal-to-noise ratio in a $1 \mathrm{~Hz}$ bandwidth of the detected signal and $\tau$ is the integration time of the measurement. Additionally, we note $N$ the detection noise power spectral density (PSD). $D$ is the discriminator slope in the error signal close to the line centre and can be 
approximated as the ratio of the signal amplitude $S$ to the linewidth of the CPT signal. From Eq. (1), the development of a stable clock requires the detection of a narrow resonance with a high signal to noise ratio.

Figure 5(a) reports the typical clock signal for an incident laser power $P_{L}$ of $475 \mu \mathrm{W}$ in the 5-cm long cell. The clock resonance is well-fitted by a lorentzian function. The CPT linewidth $\Delta \nu$ is $453 \mathrm{~Hz}$. The clock signal $S$, defined on Fig. 5(a) as $S=H-y_{0}$, is $0.104 \mathrm{~V}$. The resonance contrast $C$, defined as the ratio between the CPT signal $S$ and the dc background $y_{0}$, is $26.4 \%$. The discriminator slope $D=S / \Delta \nu$ is measured to be $2.3 \times 10^{-4} \mathrm{~V} / \mathrm{Hz}$. Figure $5(\mathrm{~b})$ shows the measured total detected noise at the output of the photodiode in four different configurations: in the dark (a), without any stabilization in presence of the CPT cell (b), with both laser frequency and AOM-based laser power servo active but without any CPT cell (c), with both laser frequency and AOM-based laser power servo active in presence of the CPT cell (d). For the latter (curve d), the detection noise is measured at half-height of the CPT resonance. The voltage noise power spectral density (PSD) at the output of the photodetector in the dark at $f=f_{m}=125 \mathrm{~Hz}$ is -149 $\mathrm{dBV}^{2} / \mathrm{Hz}$, yielding $3.54 \times 10^{-8} \mathrm{~V} / \sqrt{\mathrm{Hz}}$. Without any stabilization, the detection noise is measured to be $-120 \mathrm{dBV}^{2} / \mathrm{Hz}$ at $f=125 \mathrm{~Hz}$. With laser frequency and laser power stabilization in presence of the cell (d), the detection noise is reduced by about $8 \mathrm{~dB}$ at the value of $-128 \mathrm{dBV}^{2} / \mathrm{Hz}$, i. e $4 \times 10^{-7} \mathrm{~V} / \sqrt{\mathrm{H} z}$ at $f=125 \mathrm{~Hz}$. Consequently, from Eq. (1), the expected short-term fractional frequency stability is $\sigma_{y}(1 s) \sim \frac{453}{9.192 \times 10^{9}} \frac{4 \times 10^{-7}}{0.104} \sim 2 \times 10^{-13}$.

Figure 6 shows the measured short-term fractional frequency stability of the CPT clock. In normal operation (curve b), the latter is measured to be $3 \times 10^{-13} \tau^{-1 / 2}$ up to about 100 s averaging time, in correct agreement with SNR measurements. These short-term frequency stability performances, comparable to state-of-the-art CPT clocks [20], are very encouraging for the development of a highperformance Cs vapor cell CPT atomic clock using a single-modulated laser. The bump between 1 and $10 \mathrm{~s}$ is attributed to the EOM bias voltage servo loop gain. We suspect that this issue could prevent to observe the expected short-term stability of $2 \times 10^{-13}$. For confirmation, we performed during a shorter time a measurement of the clock frequency stability by turning off the EOM bias servo loop (Fig. 6, curve a). In this case, the bump between 1 and $10 \mathrm{~s}$ clearly disappears. For integration times higher than $10 \mathrm{~s}$, we note that the observed difference between the two stability curves are within the measurement error bars. For future long-term measurements, we prefer operating the clock with stabilized EOM bias voltage to prevent the slow increase of the optical carrier power along time [33]. After $100 \mathrm{~s}$ (see curve b), the clock frequency drifts to reach $2 \times$ $10^{-13}$ at $5000 \mathrm{~s}$. 


\section{B. Noise contributions}

Main noise sources that limit the signal to noise ratio are the photon shot noise, the detector noise, the local oscillator phase noise through the intermodulation effect $[21,22]$, the laser AM noise and the laser FM noise via the FM-AM noise conversion process in the cell.

Assuming a laser power $P_{o}$ impacting the photodetector at the output of the cell, the contribution of the photon shot noise to the clock frequency stability $\sigma_{y_{s h o t}}$ is given by [46]:

$$
\sigma_{y_{s h o t}} \sim \frac{1}{Q} \frac{1}{C \sqrt{\frac{P_{o}}{2 h \nu}}}
$$

where $Q=\nu_{0} / \Delta \nu$ is the atomic resonance Q-factor and $h \nu$ the thermal energy of a single photon. We calculate $Q=20.2 \times 10^{6}$, yielding with $P_{o} \sim 237.5 \mu \mathrm{W}, \sigma_{y_{\text {shot }}} \sim 8 \times 10^{-15}$ at $\tau=1 \mathrm{~s}$.

The detector consists of the combination of a Si photodiode (Hamamatsu S1335-66BQ) and an operational-amplifier (OP27G) based transimpedance amplifier. The feedback resistance $R$ is 52 $\mathrm{k} \Omega$ and the feedback capacitor is $22 \mathrm{pF}$. The voltage noise PSD of the feedback resistance is $4 k T R=8.5 \times 10^{-16} \mathrm{~V}^{2} / \mathrm{Hz}$, i. e $2.9 \times 10^{-8} \mathrm{~V} / \sqrt{\mathrm{Hz}}$. We also consider the detector output voltage noise $R I_{d}$ that results from the operation amplifier input current noise $I_{d}(0.6 \mathrm{pA} / \sqrt{\mathrm{Hz}})$. We find $R I_{d}=3.1 \times 10^{-8} \mathrm{~V} / \sqrt{\mathrm{Hz}}$. The operational amplifier input voltage noise is neglected. Assuming the value $D$ of the clock discriminator slope, we find that the contribution of the detector noise to the clock frequency stability is $\sigma_{y_{\text {det }}} \sim 2 \times 10^{-14}$. On Fig. 5(b) (curve a), the voltage noise PSD at the output of the photodetector in the dark at $f=125 \mathrm{~Hz}$ is $-149 \mathrm{dBV}^{2} / \mathrm{Hz}$, yielding $3.5 \times$ $10^{-8} \mathrm{~V} / \sqrt{\mathrm{Hz}}$. Knowing that the CPT resonance discriminator slope $D$ is $2.3 \times 10^{-4} \mathrm{~V} / \mathrm{Hz}$, the detector noise contribution to the clock frequency stability is measured to be $1.7 \times 10^{-14}$ at $\tau=$ $1 \mathrm{~s}$, in correct agreement with the expected value.

The short-term frequency stability of an atomic clock can be degraded by down conversion of the LO frequency noise at even multiples of the cycle rate into the frequency band of the selective resonator response. In a $\mathrm{CW}$ atomic clock with resonance frequency $\nu_{0}$, operating at a $\mathrm{LO}$ modulation frequency $f_{m}$, the frequency stability limitation $\sigma_{y_{L O}}(\tau)$ due to this aliasing effect is well approximated by [22]:

$$
\sigma_{y_{L O}}(1 s) \sim \frac{f_{m}}{\nu_{0}} \sqrt{S_{\varphi}\left(2 f_{m}\right)}
$$

where $S_{\varphi}\left(2 f_{m}\right)$ is the power spectral density (PSD) of the local oscillator phase fluctuations in the free-running regime at Fourier frequency $f=2 f_{m}$.

In our laboratory experiment, the local oscillator is a microwave frequency synthesizer driven by a 
$100 \mathrm{MHz}$ signal from a quartz oscillator. The $100 \mathrm{MHz}$ quartz oscillator is phase-locked to a highstability $10 \mathrm{MHz}$ oscillator with a servo bandwidth of about $20 \mathrm{~Hz}$. A dielectric resonator oscillator (DRO) is phase-locked to the frequency-multiplied $100 \mathrm{MHz}$ oscillator with a servo bandwidth of $400 \mathrm{kHz}$. The output $4.596 \mathrm{GHz}$ signal drives the MZ EOM to generate optical sidebands frequencysplit by $9.192 \mathrm{GHz}$. Performances of this optical beatnote that actually interrogates the atoms can be evaluated using the fast photodiode FPD. Figure 7 reports at $9.192 \mathrm{GHz}$ the absolute phase noise of the local oscillator and of the optically-carried microwave signal at the output of the EOM respectively. For $f=2 f_{m}$, except from the presence of additional spurious lines, there is no relevant phase noise degradation of the absolute phase noise between the direct synthesis output and the optical beatnote. For $f>1 \mathrm{kHz}$, the phase noise of the $9.192 \mathrm{GHz}$ optical beatnote is slightly degraded and is limited at a level of $-121 \mathrm{dBrad}^{2} / \mathrm{Hz}$ for $f>100 \mathrm{kHz}$. We confirmed that this limit is intrinsically due to the phase noise measurement setup (PNMS), more precisely by the low input power $(\sim-51 \mathrm{dBm})$ of the microwave amplifier [47] placed at the output of the fast photodiode used to detect the optical beatnote. The bump at $400 \mathrm{kHz}$ comes from the phase lock of a dielectric resonator oscillator (DRO) onto the frequency-multiplied $100 \mathrm{MHz}$ quartz oscillator. We measure $S_{\varphi}(f)=-101 \mathrm{dBrad}^{2} / \mathrm{Hz}$ at $2 f_{m}=250 \mathrm{~Hz}$. We deduce from Eq. (3) $\sigma_{y_{L O}}=1.1 \times 10^{-13}$ at $\tau=1 \mathrm{~s}$.

Last noise mechanisms are the laser AM noise and the laser FM noise through the FM-AM conversion process in the cell. On Fig. 5(b) (curve c), the CPT cell is removed and the laser power impinging on the photodetector is adjusted to be similar to the one in clock operation, in presence of the CPT cell. In this case, FM-AM conversion is canceled and the measured noise results mainly from the pure laser AM noise. In this configuration, we observe that the total detection noise is largely smaller than in presence of the CPT cell. For curve (d), the total detection noise is greatly increased with a bump at 1-2 kHz that results from the laser frequency servo loop. Consequently, we suspect that the main limitation to the clock short-term frequency stability results from FM-AM conversion process in the cell. These respective contributions will be investigated in more detail in the future. Following a methodology analog to the one described in [8], we confirm that the total laser noise is presently the main limitation to the clock short-term frequency stability at the level of $1.7 \times 10^{-13}$ at $\tau=1 \mathrm{~s}$.

A source of laser FM noise can be attributed to the laser current driver noise. The laser current driver noise was measured to be $71 \mathrm{pA} / \sqrt{\mathrm{Hz}}$ in a bandwidth of $100 \mathrm{kHz}$, yielding typical fluctuations of $22 \mathrm{nA}$ rms. Taking into account that the laser current-frequency dependence is about 1 $\mathrm{GHz} / \mathrm{mA}$, this yields a laser frequency variation of $22 \mathrm{kHz}$. We estimate that this impacts at the 
level of $1.2 \times 10^{-14}$ on the clock short-term frequency stability at $\tau=1 \mathrm{~s}$.

Table I resumes main contributions to the clock short term frequency stability at $1 \mathrm{~s}$. Main limitations are the laser AM noise, FM-AM conversion process in the cell and the local oscillator phase noise through the intermodulation effect. Other contributions are at least an order of magnitude lower and negligible at the moment.

\section{MID-TERM FREQUENCY STABILITY LIMITATIONS}

We report in this section a preliminar study to explain mid-term limitations of the clock fractional frequency stability. The clock frequency $\nu_{c}$ can be written as:

$$
\nu_{c}=\nu_{0}+S_{B}+S_{b g}+S_{l}
$$

where $S_{B}$ is the quadratic Zeeman shift, $S_{b g}$ is the collisional frequency shift due to the presence of buffer gas in the cell and $S_{l}$ is the clock frequency shift due to laser intensity or frequency variations. We investigate these different contributions in the following section.

\section{A. Second-order Zeeman shift}

The presence of a static magnetic field is expected to induce a shift of the Cs clock frequency through the Zeeman effect such as:

$$
S_{B}=\alpha_{B} B^{2}
$$

with $\alpha_{B}=427$ and $B$ in Gauss [45].

The magnetic field $B$ includes the combination of different contributions such as:

$$
B=B_{0}+B_{r}+B_{s}
$$

where $B_{0}$ is the quantization magnetic field applied by the solenoid, $B_{r}$ is the spurious residual field measured in the physics package in presence of magnetic shields when $B_{0}=0$ and $B_{s}$ is the field related to the noise of the magnetic shields [48]. The fluctuations of these terms on clock frequency can be characterized by their respective variances, $\sigma_{B_{0}}^{2}, \sigma_{B_{r}}^{2}$ and $\sigma_{B_{s}}^{2}$, such as:

$$
\sigma_{y}^{2}(\tau) \simeq\left\{\frac{2 \alpha_{B} B_{0}}{\nu_{c}}\right\}^{2}\left(\sigma_{B}^{2}\right)=\left\{\frac{2 \alpha_{B} B_{0}}{\nu_{c}}\right\}^{2}\left(\sigma_{B_{0}}^{2}+\sigma_{B_{r}}^{2}+\sigma_{B_{s}}^{2}\right)
$$


At time of experiment, we did not have in the laboratory a high-resolution gauss-meter and we were not able to measure accurately spurious field contributions. Consequently, in order to overcome this issue and to evaluate the Zeeman shift contribution, we stabilized the local oscillator frequency onto the magnetic-field sensitive $\left|F=3, m_{F}=+1\right\rangle \rightarrow\left|F=4, m_{F}=+1\right\rangle$ Zeeman transition. Figure 8 shows the typical frequency fluctuations of the local oscillator when stabilized onto this transition. The typical frequency fluctuation is measured to be at the level of $1 \mathrm{~Hz}$ for an averaging time of $1000 \mathrm{~s}$. Assuming that this transition is shifted with the static magnetic field with a sensitivity of about $700 \mathrm{kHz} / \mathrm{G}$, we deduce a typical magnetic field fluctuation at the level of $1.42 \mu \mathrm{G}$ at $\tau=1000 \mathrm{~s}$. This yields a contribution of the second-order Zeeman field to the clock fractional frequency stability at the level of $1.1 \times 10^{-14}$ at $\tau=1000 \mathrm{~s}$.

\section{B. Collisional frequency shift}

The presence of a buffer gas causes a frequency shift $S_{b g}$ of the clock frequency that can be written for a given gas in a limited temperature range as [45]:

$$
S_{b g}=P\left(\beta+\delta T+\gamma T^{2}\right)
$$

where $P$ is the buffer gas pressure at $0^{\circ} \mathrm{C}$ and $T$ is the cell temperature in ${ }^{\circ} \mathrm{C} . \beta, \delta$ and $\gamma$ are pressure, linear temperature and quadratic temperature coefficients respectively. When a mixture of two buffer gases 1 and 2 is used, Eq. (8) becomes:

$$
\Delta \nu_{b g}=P\left[\left(r_{1} \beta_{1}+r_{2} \beta_{2}\right)+\left(r_{1} \delta_{1}+r_{2} \delta_{2}\right) T+\left(r_{1} \gamma_{1}+r_{2} \gamma_{2}\right) T^{2}\right]
$$

where $\beta_{i}, \delta_{i}$ and $\gamma_{i}$ with $i=1,2$ are pressure and temperature coefficients for buffer gas 1 and 2 respectively. $r_{1}$ and $r_{2}$ denote the ratio between the buffer gas pressure and total pressure for the buffer gas 1 and buffer gas 2 respectively such as $r_{1}+r_{2}=1$.

Figure 9(a) plots the frequency shift of the clock transition $S_{b g}$ versus the cell temperature. Experimental values (filled squares) are compared to the expected frequency shift values (open circles) using coefficients reported in [49] for the present buffer-gas filled cell. For each cell temperature, the clock frequency is measured for several laser power values and linear extrapolation to null laser intensity is reported. The value of $S_{B}$ is also subtracted to isolate the value of $S_{b g}$. In the temperature range studied $\left(32\right.$ to $\left.39^{\circ} \mathrm{C}\right)$, we observe a good agreement between experience and expected values from [49]. In this temperature range, experimental data are well approximated by a linear function and the temperature sensitivity of the clock frequency is measured to be 2.53 
$\mathrm{Hz} /{ }^{\circ} \mathrm{C}$, that yields in fractional frequency to $2.8 \times 10^{-10} /{ }^{\circ} \mathrm{C}$. Figure $9(\mathrm{~b})$ shows typical temperature fluctuations of the cell sensor around a set temperature of $35.2^{\circ} \mathrm{C}$. They are measured to be $2 \times 10^{-5}{ }^{\circ} \mathrm{C}$ and about $5 \times 10^{-6}{ }^{\circ} \mathrm{C}$ at $1 \mathrm{~s}$ and $1000 \mathrm{~s}$ integration time respectively. This would yield a limitation of the clock fractional frequency stability at the level of $1.4 \times 10^{-15}$ at $\tau=1000$ S.

In Ref. [50], a simple model of the ideal gas law suggests that temperature gradients between the cell stem and the cell active volume result in buffer gas density redistribution between both cell parts and can lead to a dramatic enhanced temperature sensitivity (ETS) of the clock frequency. This geometrical effect is increased with an increased ratio $v=V_{s} / V_{c}$ between the stem volume and the cell active volume. It is also enhanced for higher temperature gradients between the stem temperature $T_{s}$ and the cell temperature $T_{c}$. In Refs $[50,51]$, the stem of the cell is outside of a microwave cavity and the ratio $v$ is about 0.03 or even higher. Up to date, we did not investigate experimentally this effect in our experiment. However, we note that in our apparatus, the cell active volume is 5 -cm long and 2-cm diameter while the cell stem is $3.5-\mathrm{cm}$ long and has an internal diameter of $1 \mathrm{~mm}$. This yields a ratio $v=0.0017$, a factor 18 lower than in the configuration reported in [51] where a ETS factor of 10 was measured. Additionally, in our experiment, the entire volume of the cell, including the stem, is inserted in a cylindrical copper cavity surrounded by a coaxial heating wire for thermal control. In permanent thermal regime, we can then think that temperature gradients between the cell volume and the stem volume are extremely small, limiting gas exchanges between both volumes. In [50], it is suggested theoretically that the ETS effect becomes negligible when the physics package is designed in such a way that $T_{s}$ and $T_{c}$ are correlated. Accordingly, we expect that this ETS effect should be greatly minimized in our experimental configuration and should not limit our performances presently. Eventually, we note that the use of a $\mathrm{N}_{2}$-Ar buffer gas mixture with a pressure ratio $r=0.56$ would allow to cancel the temperature dependence of the Cs clock frequency around the present operating temperature of about $35^{\circ} \mathrm{C}[49]$ and to reduce further the cell temperature dependence of the clock frequency.

\section{Light shift}

In the inset of Fig. 9(a), we show a typical light shift measurement for a given cell temperature of $35.6^{\circ} \mathrm{C}$. Experimental data are fitted by a linear function with a slope of $25 \mathrm{mHz} / \mu \mathrm{W}$, yielding a contribution to the clock fractional frequency stability of $2.8 \times 10^{-12} / \mu \mathrm{W}$. As shown on Fig. 10 , relative laser power fluctuations $\Delta P / P$ at the input of the cell at $\tau=1000 \mathrm{~s}$ are about 4 
$\times 10^{-5}$, that brings $\Delta P=0.019 \mu \mathrm{W}(19 \mathrm{nW})$ for a typical total laser power of $475 \mu \mathrm{W}$. This yields that the intensity light shift contribution reaches the level of $5.3 \times 10^{-14}$ at $\tau=1000 \mathrm{~s}$. This effect is presently the main limitation to the mid-term frequency stability performances of the clock. Various articles in the literature $[42,52-54]$ report the possibility to cancel the light shift slope by adjusting the power of the microwave signal driving the optical system. In these articles, optical sidebands generation was performed by directly modulating a diode laser through its injection current or by using an external phase electro-optic modulator. In our Mach-Zehnder intensity EOM based system, as reported in Fig. 11(a) for a cell temperature of $35^{\circ} \mathrm{C}$, no significant variation of the light shift slope was found by tuning the $4.596 \mathrm{GHz}$ signal power driving the EOM. This can be explained by the fact that, in our MZ EOM-based system, the optical carrier is actively rejected and changing the microwave power does not change significantly the amplitude symmetry between both first-order optical lines that participate to the CPT interaction. For additional information, we measured the evolution of the light-shift coefficient versus the cell temperature. Results are reported on Fig. 11(b). In this experiment, the light shift slope was found to decrease from about 29 to about $19 \mathrm{mHz} / \mu \mathrm{W}$ for temperatures in the $32-39^{\circ} \mathrm{C}$ range. The impact of the cell temperature on intensity-light shift has already been reported in $[54,55]$, with even a possible change of the light shift slope at a given temperature. The variation of the intensity-light shift with temperature can be explained by different mechanisms. First, increasing the cell temperature induces a broadening of optical lines (increase of the Doppler width), increasing the overlapping of optical transitions. This overlapping can induce a temperature-dependent asymetry of the CPT resonance, explaining that laser intensity-light shift can change with temperature. Second, the shift of optical lines is known to depend on the cell temperature [49], yielding a change of the optical detuning with the cell temperature and potentially a change of the intensity-light shift. Third, the absorption of the vapor is increased with temperature. This yields to a reduction of the "effective" light seen by atoms, possibly explaining a reduction of the intensity-light shift. Simultaneously, the increase of the cell absorption cause enhanced intensity gradients along the cell length. The resulting asymmetry of the CPT resonance, changing with temperature, could explain a change with temperature of intensity-light shift. A detailed study would be necessary to quantify clearly the contribution of these different mechanisms. In our experiment, we note that the light shift slope was reduced by about one order of magnitude at a temperature of about $45^{\circ} \mathrm{C}$. Nevertheless, at this temperature, the CPT signal and consequently the short-term frequency stability was degraded by a factor 2-3.

For additional information, Fig. 12(a) plots the sensitivity of the clock frequency to laser frequency 
variations. In the laser detuning range studied, experimental data are well fitted by a linear function with a coefficient of $0.039 \mathrm{~Hz} / \mathrm{MHz}$, yielding in fractional frequency $4.2 \times 10^{-12} / \mathrm{MHz}$. We measured the typical laser frequency fluctuations in clock operation by beating the laser signal with the one from another DFB laser frequency-stabilized by saturated absorption. Figure 12(b) plots the laser beat-note frequency fluctuations versus averaging time. At $1 \mathrm{~s}$ and $1000 \mathrm{~s}$, the laser beatnote frequency fluctuations are 4 and $4.7 \mathrm{kHz}$ respectively, yielding a laser beatnote relative frequency stability of $1.2 \times 10^{-11}$ and $1.4 \times 10^{-11}$ at $1 \mathrm{~s}$ and $1000 \mathrm{~s}$ respectively. These results are comparable to those reported in [35]. Assuming that both lasers contribute equally to these fluctuations, we estimate that the typical clock laser frequency fluctuations are $3.3 \mathrm{kHz}$ at $1000 \mathrm{~s}$, yielding a limitation to the clock fractional frequency stability at the level of $1.4 \times 10^{-14}$ at $\tau=$ $1000 \mathrm{~s}$

\section{Other shifts}

The impact of other experimental parameters on the clock frequency was preliminary investigated experimentally. It concerns specific components of the optics setup, including the MachZehnder electro-optic modulator (MZ EOM) and the Michelson-based system. While following results were not modelized and not explained theoretically, we considered that these results were important to be reported to estimate their contribution to the clock mid-term fractional frequency stability.

Figure 13 shows the evolution of the clock frequency versus the EOM temperature $T_{E O M}$. Experimental data are well fitted by a second order polynomial function such as $\nu_{c}-\nu_{0}(\mathrm{~Hz})=9328+4.1$ $T_{E O M}-0.05 T_{E O M}{ }^{2}$. The clock frequency sensitivity to EOM temperature variations is canceled at the first order around $T_{i}=41^{\circ} \mathrm{C}$ close to the point where the EOM transmission is maximized (see Fig. 2). The curvature $C$ around $T_{i}$ of the frequency - EOM temperature characteristic defined as $C=\frac{1}{\nu_{c}} \frac{\delta^{2} \nu_{c}}{\delta T_{E O M}^{2}}=\frac{2 \times 0.05}{\nu_{c}}$ is $1.1 \times 10^{-11} \mathrm{~K}^{-2}$. Typical EOM temperature fluctuations around the setpoint were measured to be $70 \mu \mathrm{K}$ for $\tau=1000 \mathrm{~s}$. Assuming that the temperature control system allows to set the EOM temperature $T_{E O M}$ at $T_{i} \pm 1 \mathrm{~K}$, the clock frequency stability would be limited to $7.7 \times 10^{-16}$ at $\tau=1000 \mathrm{~s}$.

A specificity of our experimental setup is the use of a Michelson delay-line system to produce the PPOP interaction scheme. One arm of the Michelson system is fixed. In the second arm, the mirror is placed on a translation axis to evaluate the sensitivity of the clock frequency to variations of the optical path difference between both arms of the interferometer. Figure 14 shows the 
evolution of the clock frequency versus the mobile mirror position $x$. Experimental data are well fitted by a second order polynomial function such as $\nu_{c}-\nu_{0}(\mathrm{~Hz})=9109+17.2 x-0.35 x^{2}$. The clock frequency sensitivity to the mirror position translation is canceled at the first order around $x_{i}=24.3 \mathrm{~mm}\left(\simeq 3 \frac{\lambda_{c s}}{4}\right)$ where the CPT signal is maximized [33]. The curvature $C$ around $x_{i}$ of the frequency-mirror position characteristic is $C=\frac{1}{\nu_{c}} \frac{\delta^{2} \nu_{c}}{\delta x^{2}}=3.8 \times 10^{-11} \mathrm{~mm}^{-2}$. Presently, no active servo is implemented to reduce mechanical vibrations of the system. We estimate that the contribution of this effect to the clock fractional frequency stability is lower than $1 \times 10^{-14}$ at $\tau$ $=1000 \mathrm{~s}$.

\section{E. Resume}

Table II resumes main contributions to the clock mid-term frequency stability at $\tau=1000 \mathrm{~s}$. The latter is strongly limited by the laser intensity through the light shift effect. Other relevant mechanisms could be the laser frequency variations, the Zeeman shift and length fluctuations of the Michelson delay-line system.

\section{DISCUSSIONS}

This short section aims to discuss about potential ways to improve the performances of this CPT clock. The intermodulation effect is found to be an important contribution to the clock short-term noise budget. A low phase noise microwave synthesizer based on a high-performance $100 \mathrm{MHz}$ oven-controlled quartz oscillator (OCXO) will be implemented nextly on the experiment [56]. This source exhibits an absolute phase noise of $-106 \mathrm{dBrad}^{2} / \mathrm{Hz}$ at $f=2 f_{m}=224 \mathrm{~Hz}$ and should allow to reduce the intermodulation effect contribution at the level of about $6 \times 10^{-14}$ at $\tau=1 \mathrm{~s}$.

Laser power fluctuations are found to be a strong limitation to the clock mid-term frequency stability. A proposal to reduce laser intensity effects using a similar setup is to use a Ramseytype temporal pulsed interrogation [34]. It has been demonstrated that pulsed interaction reduces strongly the intensity-light shift sensitivity $[8,18]$, prevents the CPT line broadening and allows the detection of narrow and high-contrast Ramsey fringes if combined with optimized CPT pumping schemes $[16,30,34]$. Additionally, other light-dependent effects such as the impact of the optical sidebands asymetry, the dependence on the cell temperature of the light shift, should be further studied [55]. 
Another way to improve the mid and long term frequency stability of the PPOP Cs CPT clock is the use of a vapor cell with optimized $\mathrm{N}_{2}$ - Ar buffer gas mixture for reduced temperature sensitivity of the clock frequency. From $[49,55]$, a pressure ratio $r=P_{A r} / P_{N_{2}}=0.56$ should allow to shift the inversion temperature close to $35^{\circ} \mathrm{C}$.

\section{CONCLUSIONS}

We implemented a CW-regime CPT-based Cs vapor cell atomic clock using the push-pull optical pumping technique. The optics part of the clock combines a single diode laser, a Mach-Zehnder electro-optic modulator, an acousto-optic modulator for laser power stabilization and a Michelson delay-line system. An encouraging short-term frequency stability of $3 \times 10^{-13} \tau^{-1 / 2}$ up to $\tau=$ $100 \mathrm{~s}$ was demonstrated, in correct agreement with the signal-to-noise ratio limit. The short-term frequency stability is mainly limited by the laser AM noise, FM-AM conversion noise process and the intermodulation effect due to the local oscillator phase noise. A preliminar study to explain limitations of the clock performances at $\tau=1000 \mathrm{~s}$ was reported. Laser intensity effects were found to be a strong limitation to the clock mid-term performances.

\section{Acknowledgments}

This work has been funded by the EMRP program (IND55 Mclocks). The EMRP is jointly funded by the EMRP participating countries within EURAMET and the European Union. This work was partly supported by LNE, LabeX FIRST-TF and ANR-DGA ISIMAC project (ANR-11ASTR-0004). The authors thank deeply S. Guérandel, E. De Clercq and P. Yun (LNE-SYRTE) for supply of the CPT cell, support for computer control of the experiment and careful reading of the manuscript.

[1] R. T. Dupuis, T. Lynch and J. R. Vacaro, Proceedings of the 2008 IEEE International Frequency Control Symposium edited by Jadusliwer B. (Honolulu, Hawaii-USA) 2008, pp. 655-660.

[2] P. Waller et al., IEEE Trans. Ultrason. Ferroelec. Freq. Control 57, 3, 738 (2010).

[3] Glonass Navigation Satellite System, interface controcl document, Edition 5.1, Moscow (2008).

[4] J. Vanier and C. Mandache, Appl. Phys. B Lasers Opt. 85, DOI: 10.1007/s00340-007-2643-5 (2007).

[5] J. Camparo, The rubidium atomic clock and basic research, Phys. Today 60, 11, 33 (2007).

[6] J. Vanier, Appl. Phys. B Lasers Opt. 81, DOI: 10.1007/s00340-005-1905-3 (2005). 
[7] A. Godone, S. Micalizio, F. Levi and C. Calosso, Phys. Rev. A 74, 043401 (2006).

[8] S. Micalizio, C. E. Calosso, A. Godone and F. Levi, Metrologia 49, 425-436 (2012).

[9] M. Zhu, High contrast signal in a coherent population trapping based atomic frequency standard application, Proceedings of the 2003 IEEE International Frequency Control Symposium and PDA Exhibition Jointly with the 17th European Frequency and Time Forum, Tampa, Florida, pp 16-21, 2003.

[10] J. Lin, J. Deng, Y. Ma, H. He and Y. Wang, Opt. Lett. 37, 24, 5036-5038 (2012).

[11] C. Stefanucci, T. Bandi, F. Merli, M. Pellaton, C. Affolderbach, G. Mileti and A. K. Skrivervik, Rev. Sci. Instrum. 83, 104706 (2012).

[12] S. Kang, M. Gharavipour, C. Affolderbach, F. Gruet and G. Mileti, Journ. Appl. Phys. 117, 104510 (2015).

[13] G. Alzetta, A. Gozzini, L. Moi and G. Orriols, Nuovo Cimento B 36, 5 (1976).

[14] E. Arimondo, Coherent population trapping in laser spectroscopy, in Progress in Optics, vol. 35. New York, NY, USA: American Elsevier, 1996, pp. 257-353.

[15] A. Godone, S. Micalizio and F. Levi, Phys. Rev. A 70, 023409 (2004).

[16] T. Zanon, S. Guérandel, E. de Clercq, D. Holleville, N. Dimarcq and A. Clairon, Phys. Rev. Lett. 94, $193002(2005)$.

[17] N. F. Ramsey, Phys. Rev. 78, 695 (1950).

[18] N. Castagna, R. Boudot, S. Guérandel, E. De Clercq, N. Dimarcq, and A. Clairon, IEEE Trans. Ultrason. Ferroelec. Freq. Contr. 56, 2, 246 (2009).

[19] R. Boudot, S. Guérandel, E. De Clercq, N. Dimarcq, and A. Clairon, IEEE Trans. Instrum. Meas. 58, 4, 1217 (2009).

[20] J. M. Danet, M. Lours, S. Guérandel and E. De Clercq, IEEE Trans. Ultrason. Ferroelec. Freq. Contr. 61, 4, 567 (2014).

[21] G. J. Dick, J. D. Prestage, C. A. Greenhall, and L. Maleki, Local oscillator induced degradation of medium-term stability in passive atomic frequency standards, in Proc. 22nd Precise Time and Time Interval (PTTI) Applications and Planning Meeting, 1990, pp. 487-508.

[22] C. Audoin, V. Candelier and N. Dimarcq, IEEE Trans. Instrum. Meas. 40, 2, 121 (1991).

[23] Y. Zhang, S. Qu and S. Gu, Opt. Express. 20, 6, 6400 (2012).

[24] P. Yun, B. Tan, W. Deng, J. Yang and S. Gu, Rev. Sci. Instr. 83, 093111 (2012).

[25] B. Tan, P. Yun, J. Yang, Y. Tian and S. Gu, Appl. Phys. Lett. 102, 161117 (2013).

[26] J. Yang, Y. Tian, B. Tan, P. Yun and S. Gu, Journ. Appl. Phys. 115, 093109 (2014).

[27] Y. Y. Jau, E. Miron, A. B. Post, N. N. Kuzma and W. Happer, Phys. Rev. Lett. 93, 160802 (2004).

[28] S. A. Zibrov, V. L. Velichansky, A. S. Zibrov, A. V. Taichenachev and V. I. Yudin, JETP Lett. 82, 8, $534(2005)$.

[29] V. I. Yudin, A. V. Taichenachev, D. I. Sevostianov, V. L. Velichansky, V. V. Vasiliev, A. A. Zibrov, A. S. Zibrov and S. A Zibrov, Phys. Rev. A 87, 063806 (2013). 
[30] P. Yun, J. M. Danet, D. Holleville, E. De Clercq and S. Guérandel, Appl. Phys. Lett. 105, 231106 (2014).

[31] http://www.inrim.it/Mclocks

[32] E. Breschi, S. D. Gruji, P. Knowles and A. Weis, Appl. Phys. Lett. 104, 023501 (2014).

[33] X. Liu, J. M. Mérolla, S. Guérandel, C. Gorecki, E. De Clercq and R. Boudot, Phys. Rev. A 87, 013416 (2013).

[34] X. Liu, S. Guérandel, J. M. Mérolla, E. De Clercq and R. Boudot, Opt. Express 21, 10, 12451 (2013).

[35] X. Liu and R. Boudot, IEEE Trans. Instr. Meas. 61, 10, 2852 (2012).

[36] R. Boudot, S. Guérandel and E. De Clercq, IEEE Trans. Instr. Meas. 58, 10, 3659 (2009).

[37] R. H. Dicke, Phys. Rev. 18, 472 (1953).

[38] G. A. Pitz, D. E. Wertepny and G. P. Perram, Phys. Rev. A 80, 062718 (2009).

[39] W. Demtröder, "Laser Spectrocopy: Basic concepts and instrumentation - Third edition," SpringerVerlag ISBN 1439-26\%4, (2002).

[40] O. Schmidt, K.-M. Knaak, R. Wynands and D. Meschede, Appl. Phys. B 59, 167 (1994).

[41] S. Knappe, J. Kitching, L. Hollberg and R. Wynands, Appl. Phys. B Lasers Opt. 74, 217 (2002).

[42] R. Boudot, P. Dziuban, M. Hasegawa, R. K. Chutani, S. Galliou, V. Giordano and C. Gorecki, Journ. Appl. Phys. 109, 014912 (2011).

[43] http://www.t4science.com/documents/iMaser-Clock-Spec.pdf (iMaser-ST 3000 specifications)

[44] V. Giordano, S. Grop, B. Dubois, P. Y. Bourgeois, Y. Kersalé, G. Haye, V. Dolgovskiy, N. Bucalovic, G. Di Domenico, S. Schilt, J. Chauvin, D. Valat and E. Rubiola, Rev. Sci. Instr. 83, 085113 (2012).

[45] J. Vanier and C. Audoin, The quantum physics of atomic frequency standards, Adam-Hilger, Bristol (1989).

[46] V. Shah and J. Kitching "Advances in Coherent Population Trapping for Atomic Clocks," Advances in Atomic, Molecular and Optical Physics, Elsevier, 59, Chapter 2 (2010).

[47] R. Boudot and E. Rubiola, Phase noise in RF and microwave amplifiers, IEEE Trans. Ultrason. Ferroelec. Freq. Contr. 59, 12, 2613 (2012).

[48] T. W. Kornack, S. J. Smullin, S. K. Lee and M. V. Romalis, Appl. Phys. Lett. 90, 223501 (2007).

[49] O. Kozlova, S. Guérandel and E. De Clercq, Phys. Rev. A 83, 062714 (2011).

[50] C. E. Calosso, A. Godone, F. Levi and S. Micalizio, IEEE Trans. Ultrason. Ferroelec. Freq. Contr. 59, 12,2646 (2012).

[51] T. Bandi, C. Affolderbach, C. Stefanucci, F. Merli, A. K. Skrivervik and G. Mileti, IEEE Trans. Ultrason. Ferroelec. Freq. Contr. 61, 11, 1769 (2012).

[52] M. Zhu and L. S. Cutler, Theoretical and experimental study of light-shift in a CPT-based Rb vapor cell frequency standard, Proc. $32^{t h}$ Annual Precise Time and Time Interval (PTTI) Meeting, Reston, VA, USA, pp. 311-323 (2000).

[53] V. Shah, V. Gerginov, P. D. D. Schwindt, S. Knappe, L. Hollberg and J. Kitching, Appl. Phys. Lett. 89, 151124 (2006). 
[54] D. Miletic, C. Affolderbach, M. Hasegawa, R. Boudot, C. Gorecki and G. Mileti, Appl. Phys. B Lasers Opt. 109, 1, 89 (2012).

[55] O. Kozlova, J. M. Danet, S. Guérandel and E. De Clercq, IEEE Trans. Instr. Meas. 63, 7, 1863 (2014).

[56] B. François, C. E. Calosso, J. M. Danet and R. Boudot, Rev. Sci. Instr. 85, 094709 (2014). 


\section{Table captions}

1. Main contributions to the clock short term frequency stability at $\tau=1 \mathrm{~s}$.

2. Main contributions to the clock mid-term frequency stability at $\tau=1000 \mathrm{~s}$. 


\section{Figure captions}

1. Schematic of the Cs vapor cell atomic clock based on push-pull optical pumping. DFB: Distributed feedback diode laser, FC: fiber collimator, MZ EOM: Mach-Zehnder electrooptic modulator, AOM: acousto-optic modulator, LO: microwave local oscillator, FPD: fast photodiode, PD: photodiode, RF: radiofrequency source, $V_{\text {ref }}$ : reference voltage, Michelson: Michelson-type delay-line and polarization orthogonalizer system, PC-DAQ: personal computer - data acquisition card. The inset shows the CPT diagram involved in the push-pull optical pumping technique.

2. Transmission of the MZ EOM versus the EOM temperature. The $4.596 \mathrm{GHz}$ microwave signal that feeds the EOM is activated.

3. Optical absorption lines detected in the Cs annex cell used for laser frequency stabilization (a) and in the buffer-gas filled CPT cell (b). For each cell, both absorption lines of the doublet are separated by $1.16 \mathrm{GHz}$. For each cell, the doublet is detected between the two doublets $\left(F=3 \rightarrow F^{\prime}=3,4\right.$ and $\left.F=4 \rightarrow F^{\prime}=3,4\right)$ of the Cs $D_{1}$ line structure. Compared to the Cs reference cell, optical lines are frequency-shifted by about $-122 \mathrm{MHz}$ in the buffer-gas filled CPT cell.

4. Zeeman spectrum in a 2-cm long buffer-gas filled cell. Comparison between circular polarization and the PPOP scheme. The $0-0$ clock transition is greatly favored with the PPOP scheme. The laser power incident in the cell is $400 \mu \mathrm{W}$. The static magnetic field is 24.5 $\mathrm{mG}$. The cell temperature is $38^{\circ} \mathrm{C}$.

5. (a): Clock CPT resonance signal. Experimental data of the direct CPT resonance are fitted by a lorentzian fit function. The total laser power incident on the cell is $475 \mu \mathrm{W}$. The CPT linewidth is $453 \mathrm{~Hz}$. The resonance contrast is $26.4 \%$. (b): Total detected noise at the output of the photodiode in different configurations. The voltage noise is measured at halfheight of the CPT resonance by shifting the local oscillator central frequency of $\Delta \nu / 2 \sim 226$ Hz. (a) in the dark (b) without laser frequency stabilization neither laser power stabilization (c) with laser power and frequency stabilization without the CPT cell (d) with laser power and frequency stabilization in presence of the CPT cell. The static magnetic field is $89 \mathrm{mG}$.

6. Allan deviation of the clock frequency. The laser power incident on the cell is $475 \mu \mathrm{W}$. The static magnetic field is $89 \mathrm{mG}$. (a): EOM bias servo loop off, (a): EOM bias servo loop 
activated. The first dashed line is a curve with a $3 \times 10^{-13} \tau^{-1 / 2}$ slope. The second dashed line is a linear curve with a slope of about $4 \times 10^{-17} \tau$ slope.

7. Absolute phase noise of the $9.192 \mathrm{GHz}$ signal. (a) direct synthesis output, (b) $9.192 \mathrm{GHz}$ optical beatnote at the output of the EOM. For information, the part of the spectrum with a $f^{-3}$ slope is shown.

8. Clock frequency fluctuations versus the integration time when the local oscillator is stabilized onto the $\left|F=3, m_{F}=+1\right\rangle \rightarrow\left|F=4, m_{F}=+1\right\rangle$ Zeeman transition. The static magnetic field is $89 \mathrm{mG}$.

9. (a): Collisional frequency shift versus the cell temperature. Empty circles are expected values of the frequency shift using coefficients reported in [49]. Filled squares are experimental data. These data are fitted in a limited temperature range by a linear function. The inset is an example for a cell temperature of $35.6^{\circ} \mathrm{C}$ of the evolution of the clock frequency versus the laser power. Extrapolation at null laser power is used to extract the collisional frequency shift. (b): Cell sensor temperature fluctuations versus the integration time $\tau$.

10. Relative laser power fluctuations versus the averaging time $\tau$.

11. (a): Light shift coefficient in $\mathrm{mHz} / \mu \mathrm{W}$ versus the microwave power at fixed cell temperature. A solid line is added to show that the light shift coefficient does not change significantly with the microwave power. (b): Light shift coefficient in $\mathrm{mHz} / \mu \mathrm{W}$ versus the cell temperature at fixed microwave power.

12. (a): Clock frequency versus the laser frequency detuning. Experimental data are fitted by a linear function. (b): Frequency fluctuations of the laser beatnote versus the averaging time $\tau$.

13. Clock frequency shift versus the EOM temperature. Squares: Experimental data. The solid line is a second-order polynomial fit function to the data.

14. Clock frequency versus the mobile mirror position. The x-axis indicates the length difference between both arms of the Michelson system. Squares: Experimental data. The solid line is a second-order polynomial fit to the data. 
TABLE I: Main contributions to the clock short term frequency stability at $\tau=1 \mathrm{~s}$.

\begin{tabular}{|c|c|}
\hline Noise Source & $\sigma(1 \mathrm{~s})$ \\
\hline Shot noise & $8 \times 10^{-15}$ \\
Detector noise & $2 \times 10^{-14}$ \\
LO phase noise & $1.1 \times 10^{-13}$ \\
Total laser noise (AM and FM-AM) & $1.7 \times 10^{-13}$ \\
Laser current driver noise & $1.2 \times 10^{-14}$ \\
Total $\sigma_{y}(1 s)=\sqrt{\sum \sigma_{y}^{2}}$ & $2 \times 10^{-13}$ \\
\hline
\end{tabular}


TABLE II: Main contributions to the clock mid-term frequency stability at $\tau=1000 \mathrm{~s}$.

\begin{tabular}{|c|c|}
\hline Physical effect & $\sigma_{y}(1000 \mathrm{~s})$ \\
\hline Magnetic field & $1.1 \times 10^{-14}$ \\
Cell temperature & $1.4 \times 10^{-15}$ \\
Laser power & $5.3 \times 10^{-14}$ \\
Laser frequency & $1.4 \times 10^{-14}$ \\
EOM temperature & $7.7 \times 10^{-16}$ \\
Mirror position & $<1 \times 10^{-14}$ \\
Total $\sigma_{y}(1000 s)=\sqrt{\sum \sigma_{y}^{2}}$ & $5.6 \times 10^{-14}$ \\
\hline
\end{tabular}




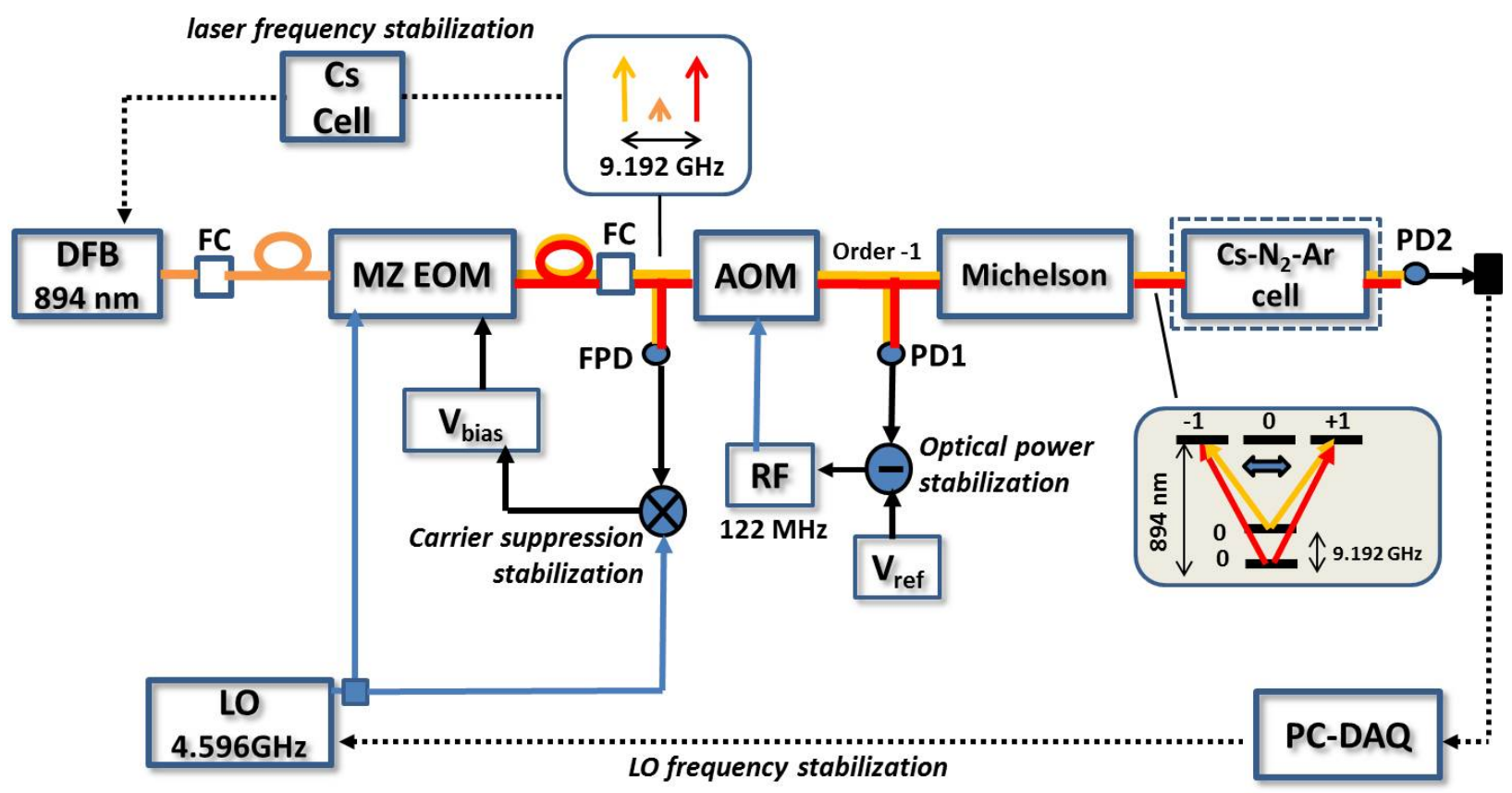

FIG. 1: 


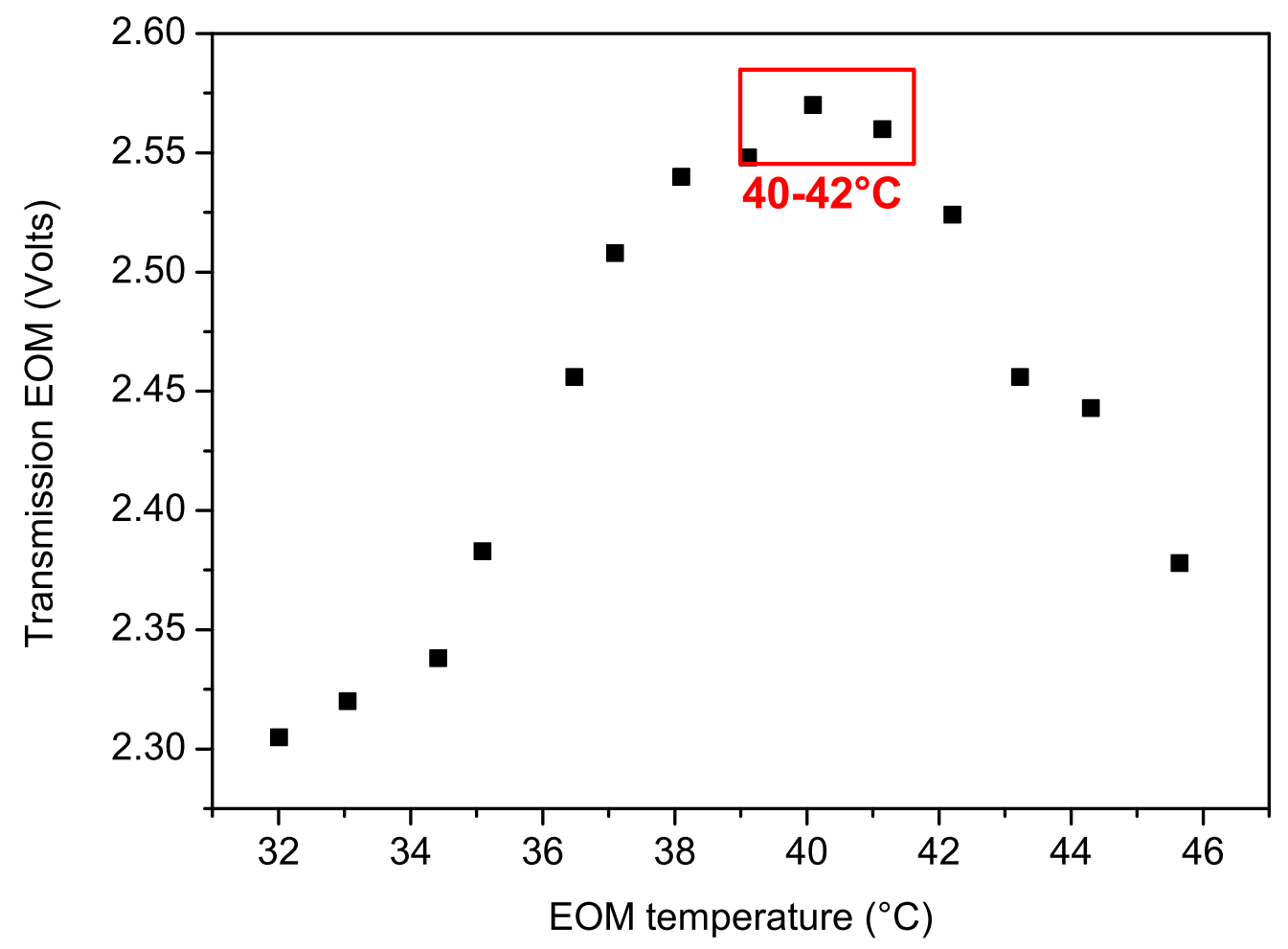

FIG. 2: 


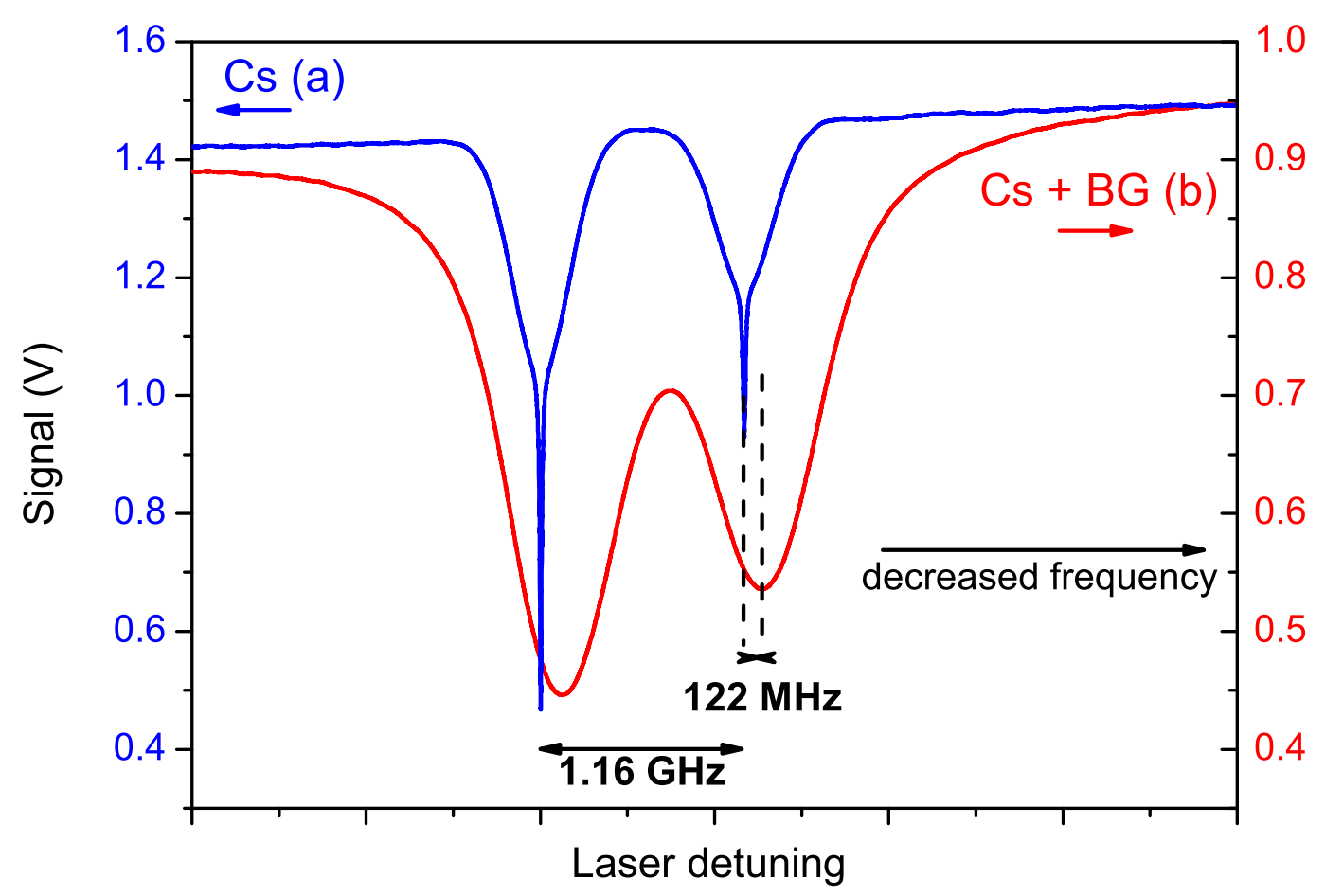

FIG. 3: 


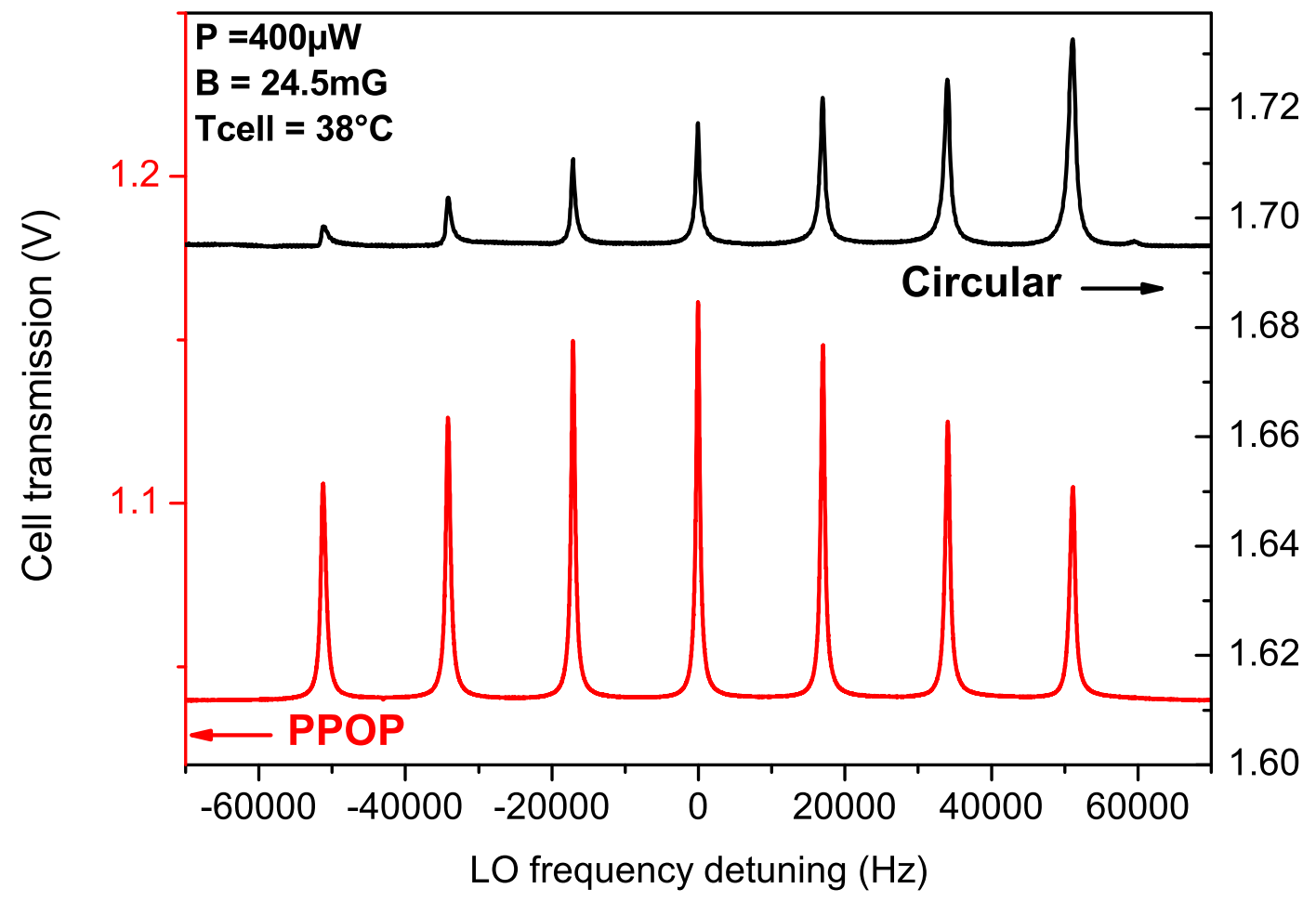

FIG. 4: 


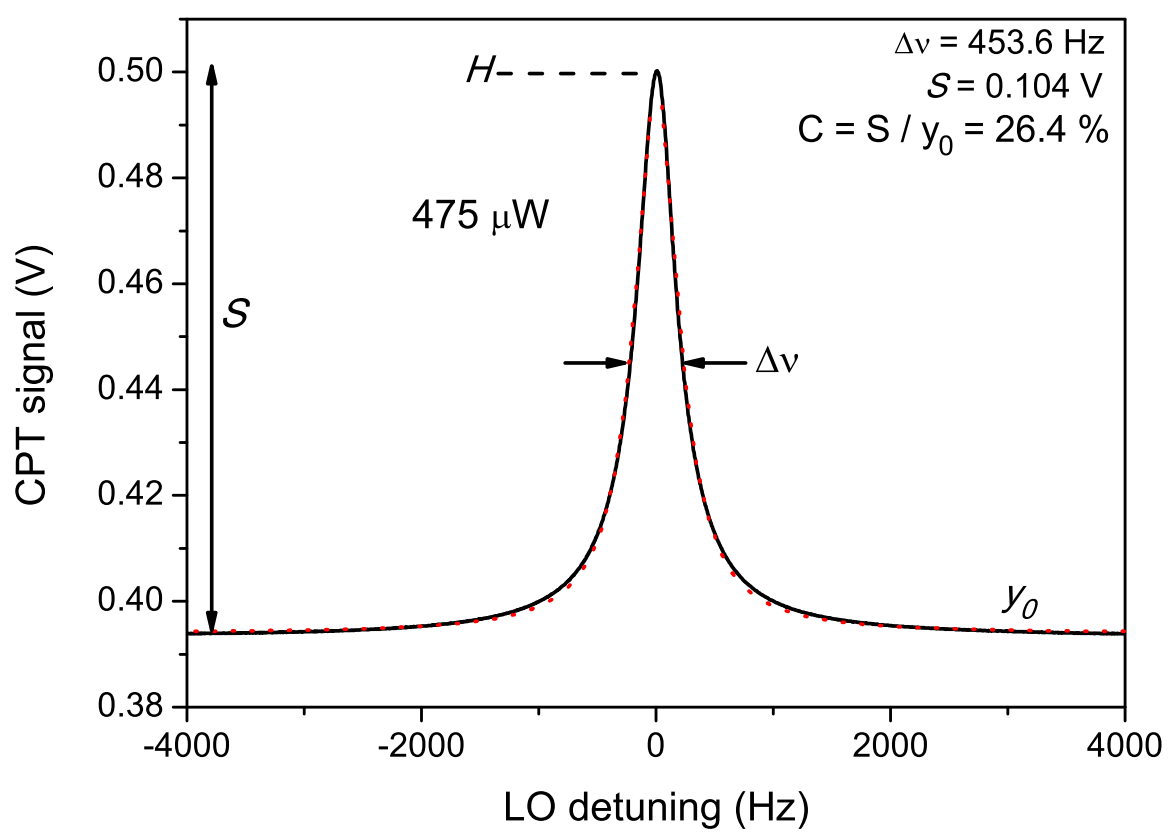

(a)

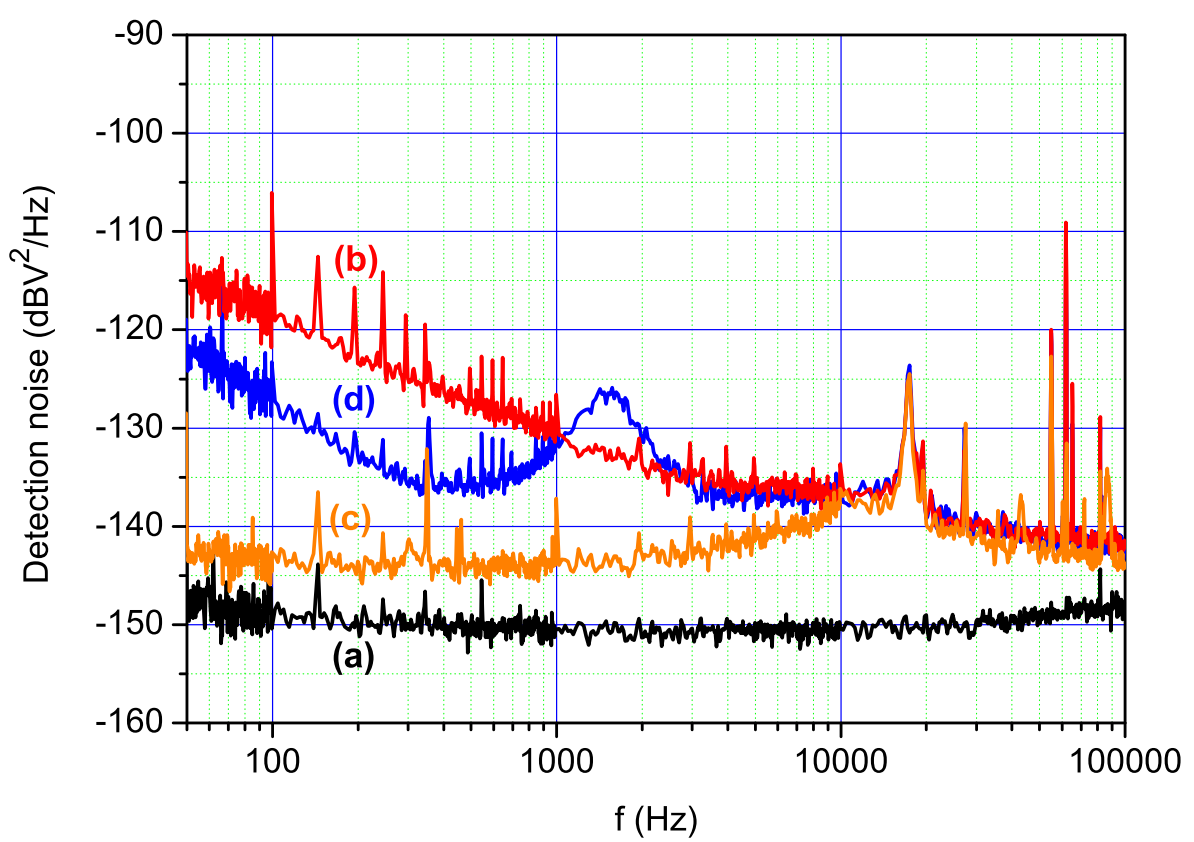

(b)

FIG. 5: 


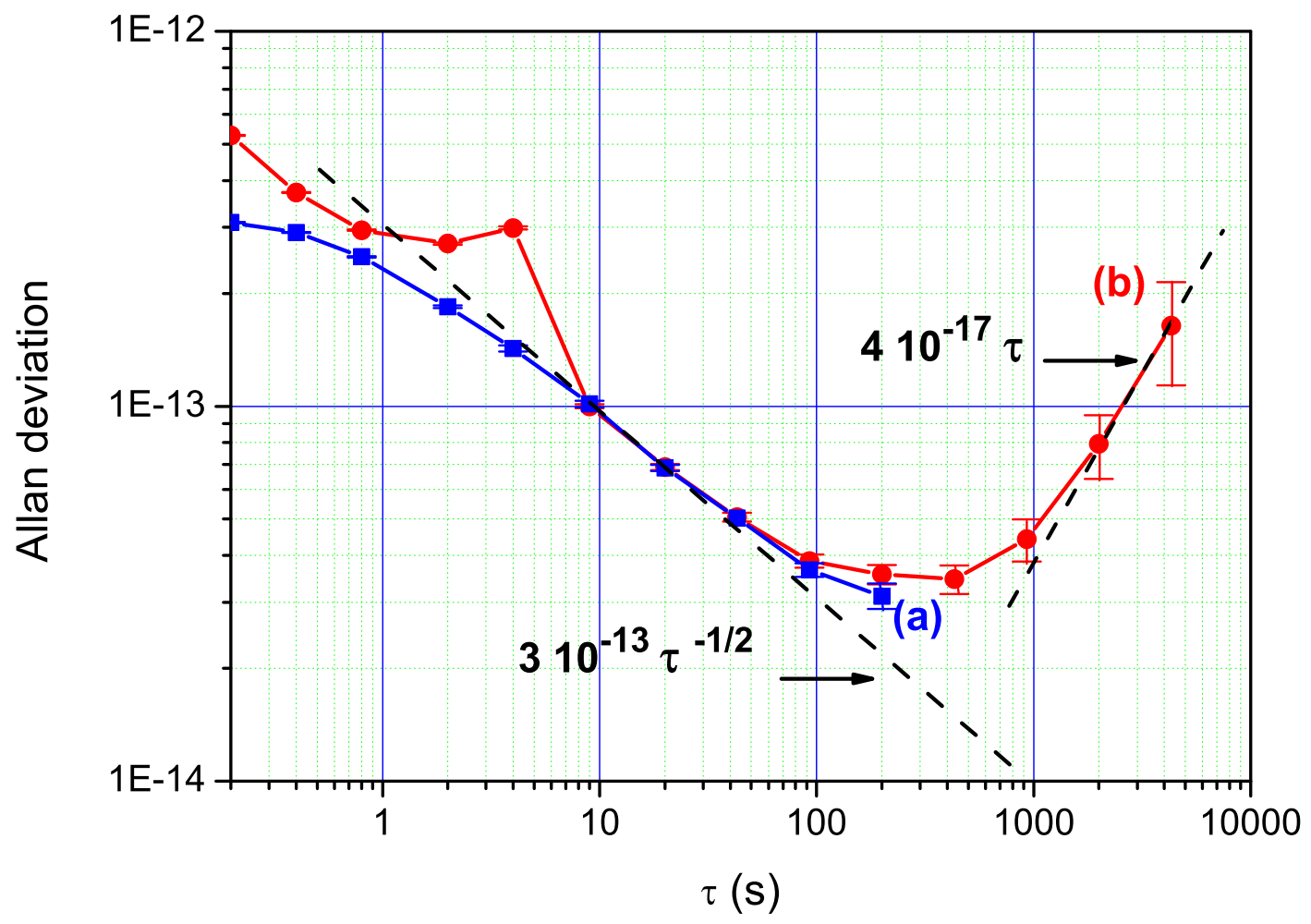

FIG. 6: 


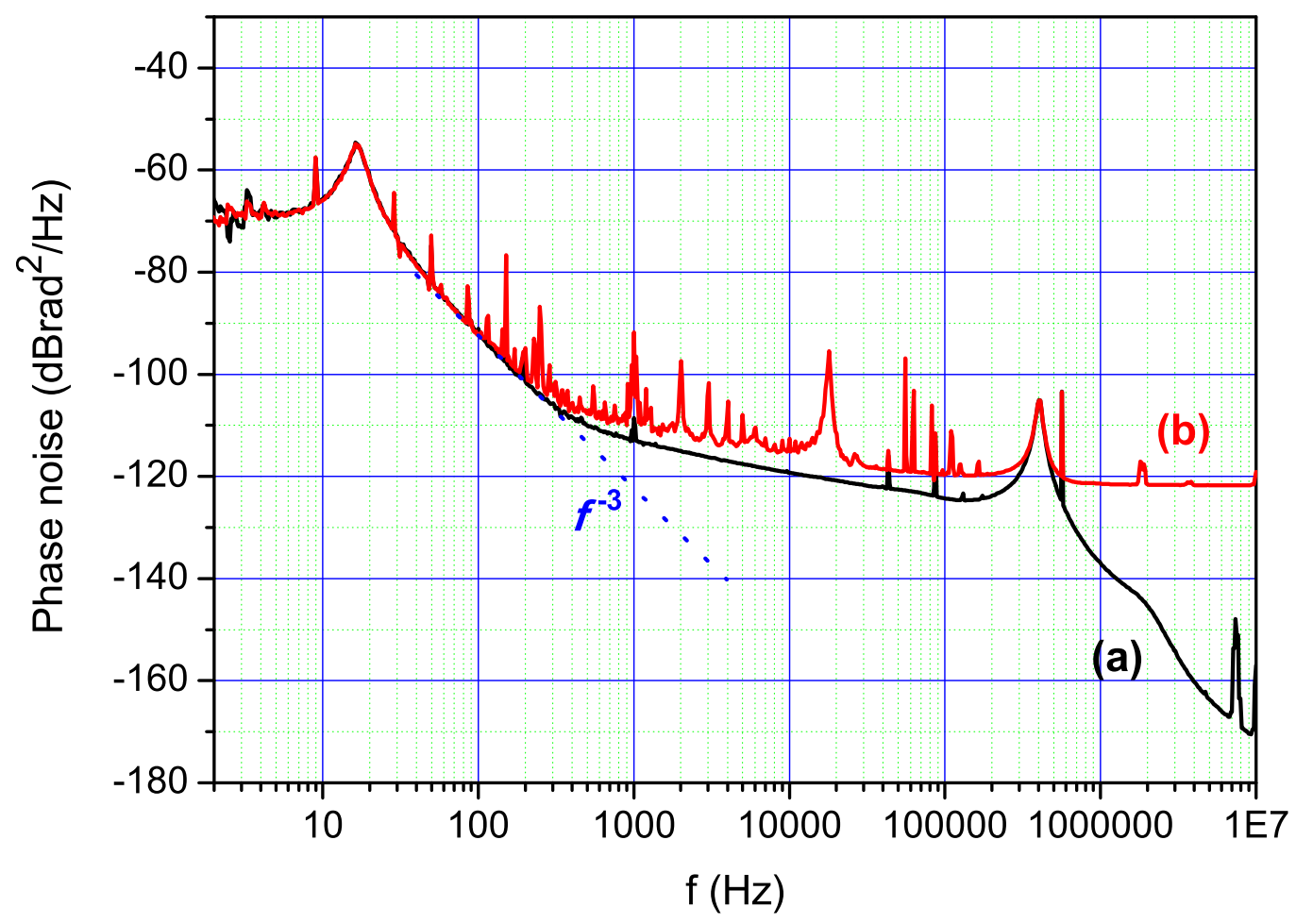

FIG. 7: 


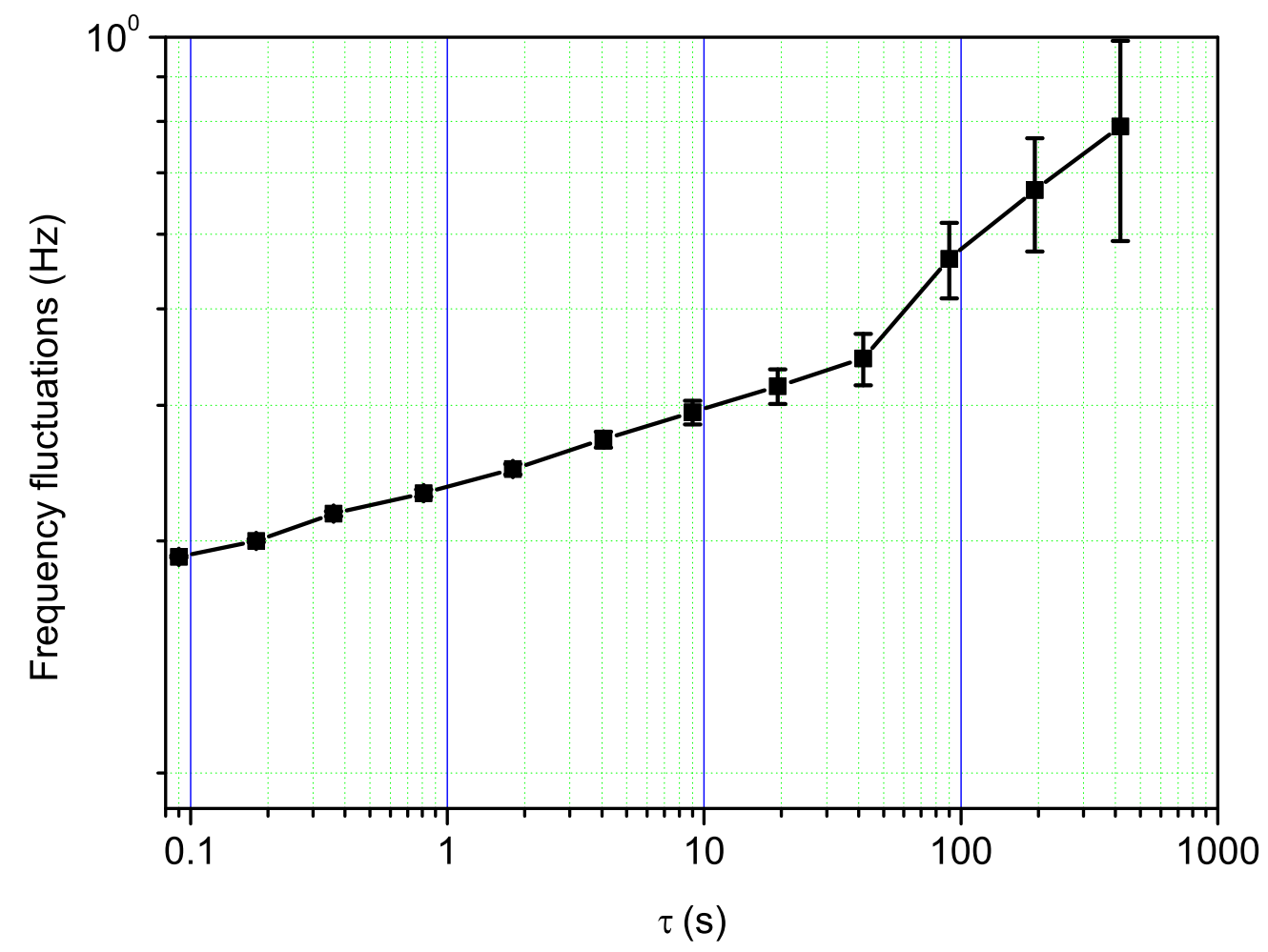

FIG. 8: 


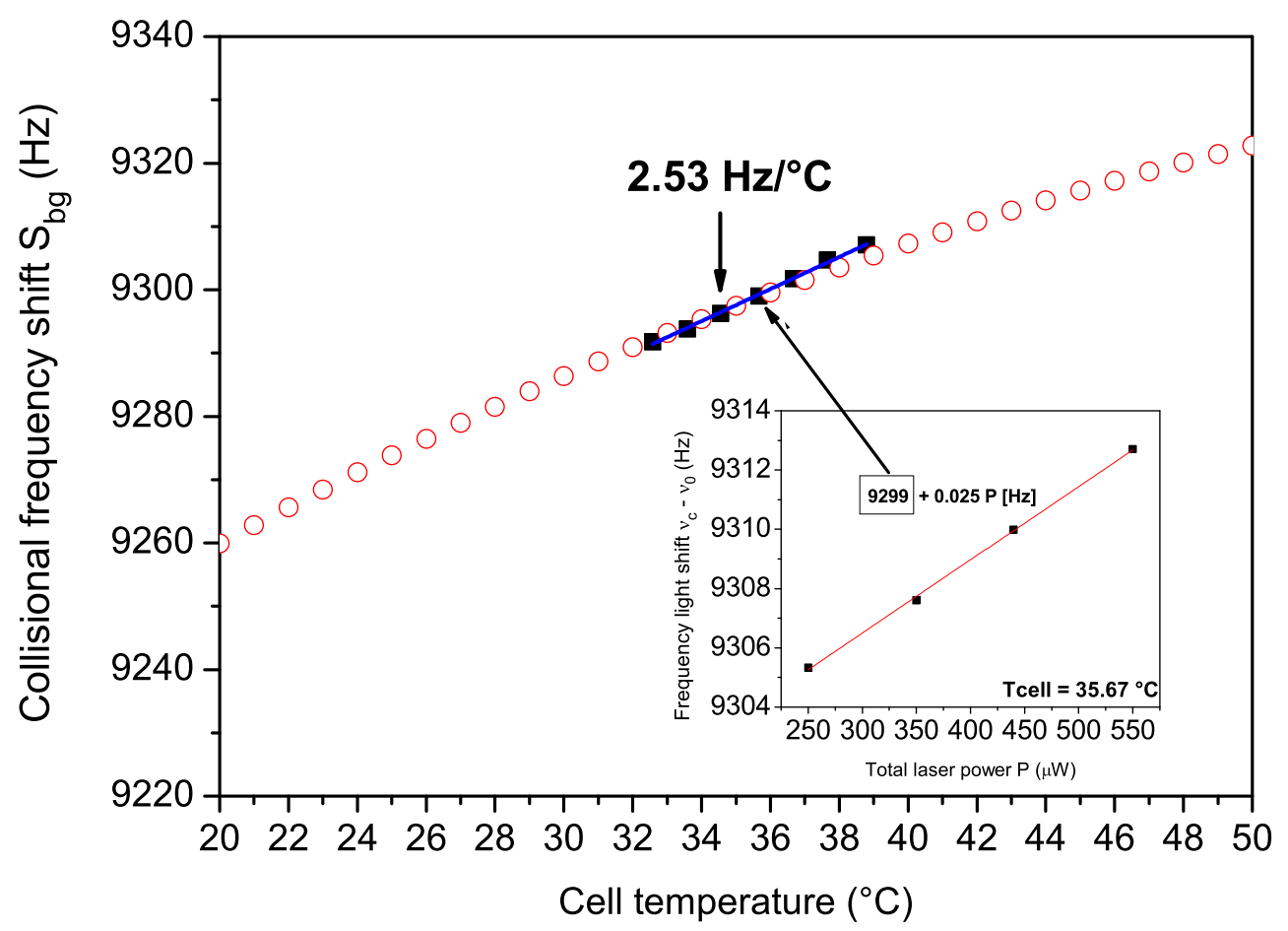

(a)

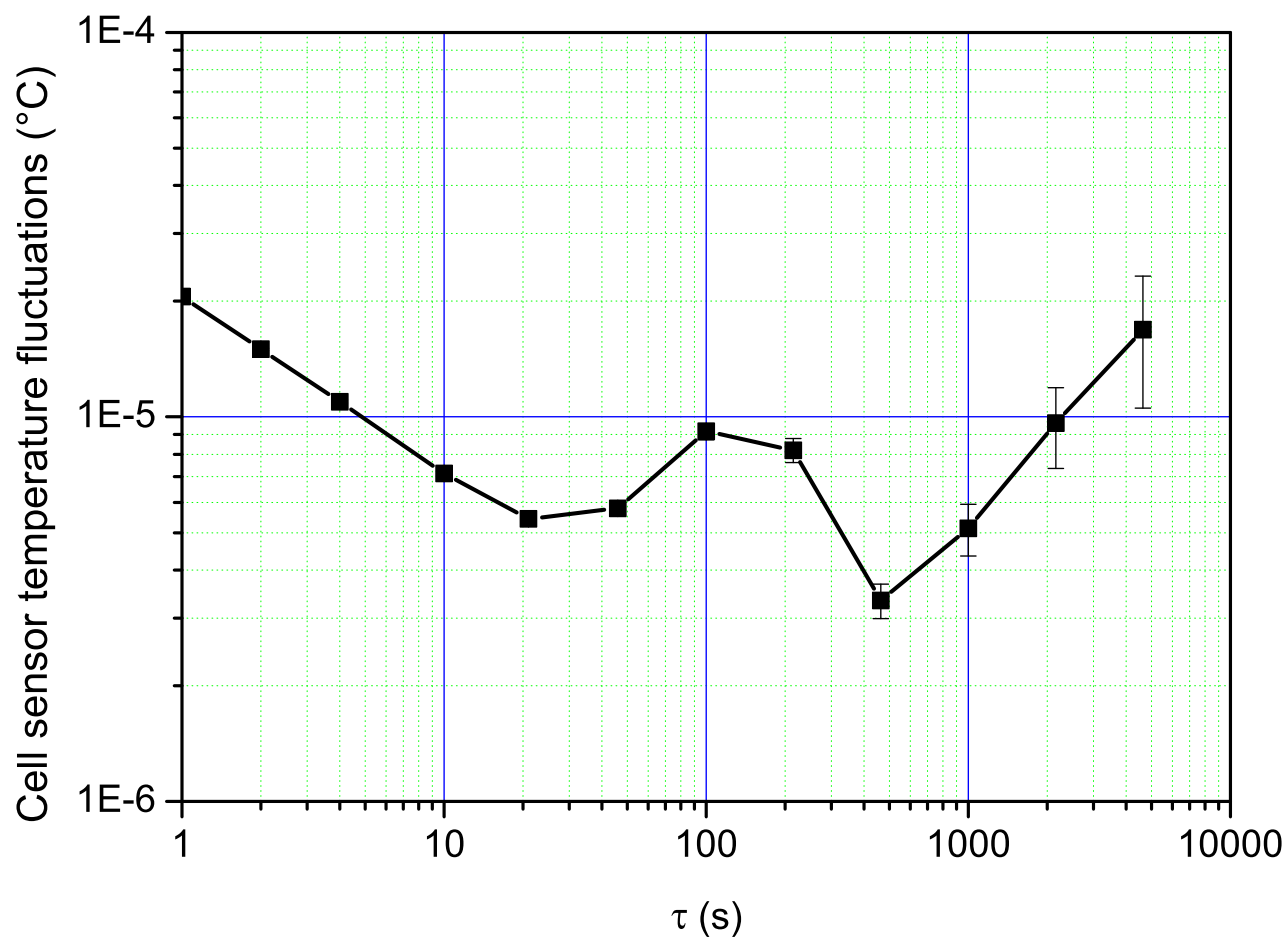

(b)

FIG. 9: 


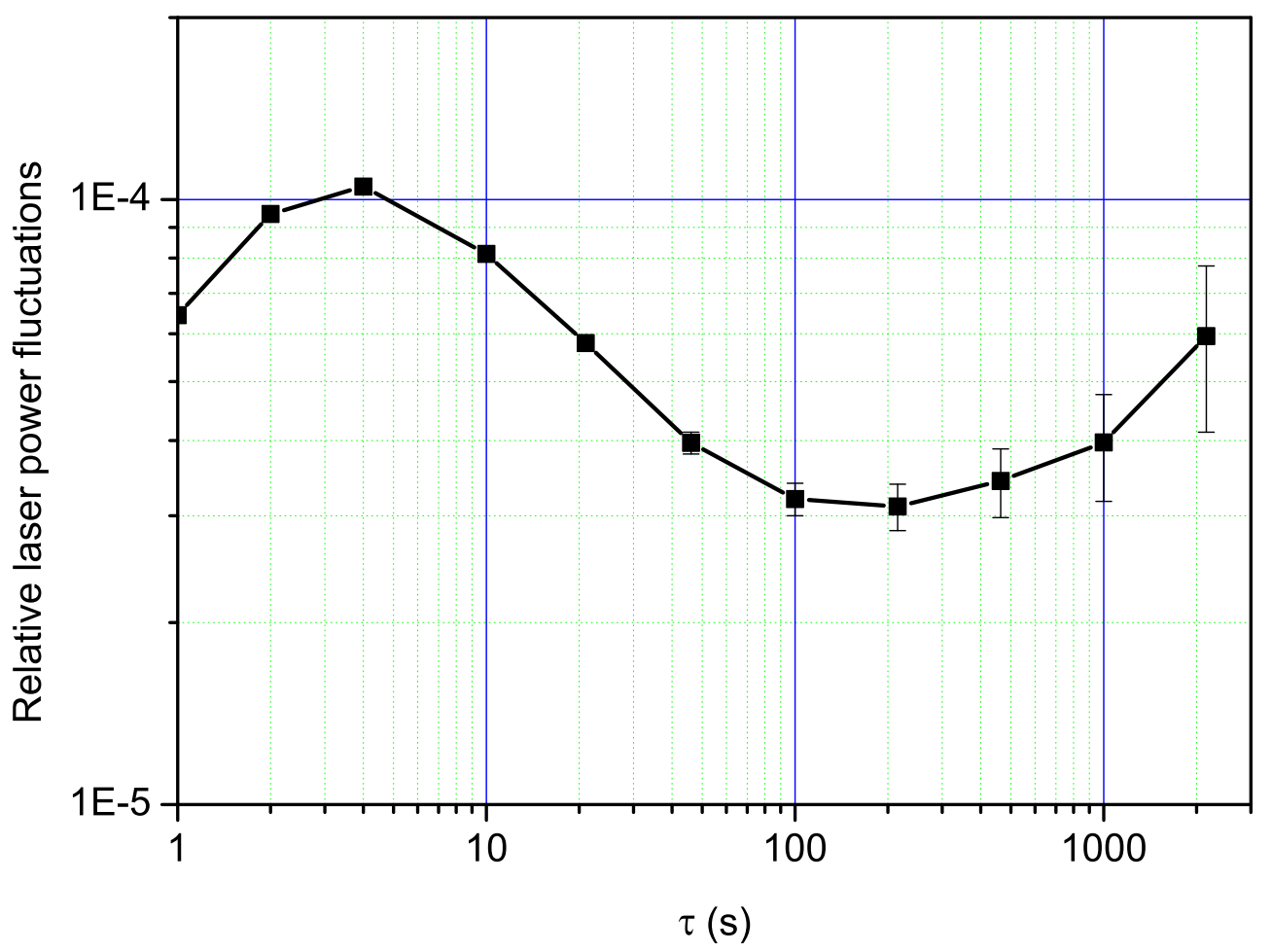

FIG. 10: 


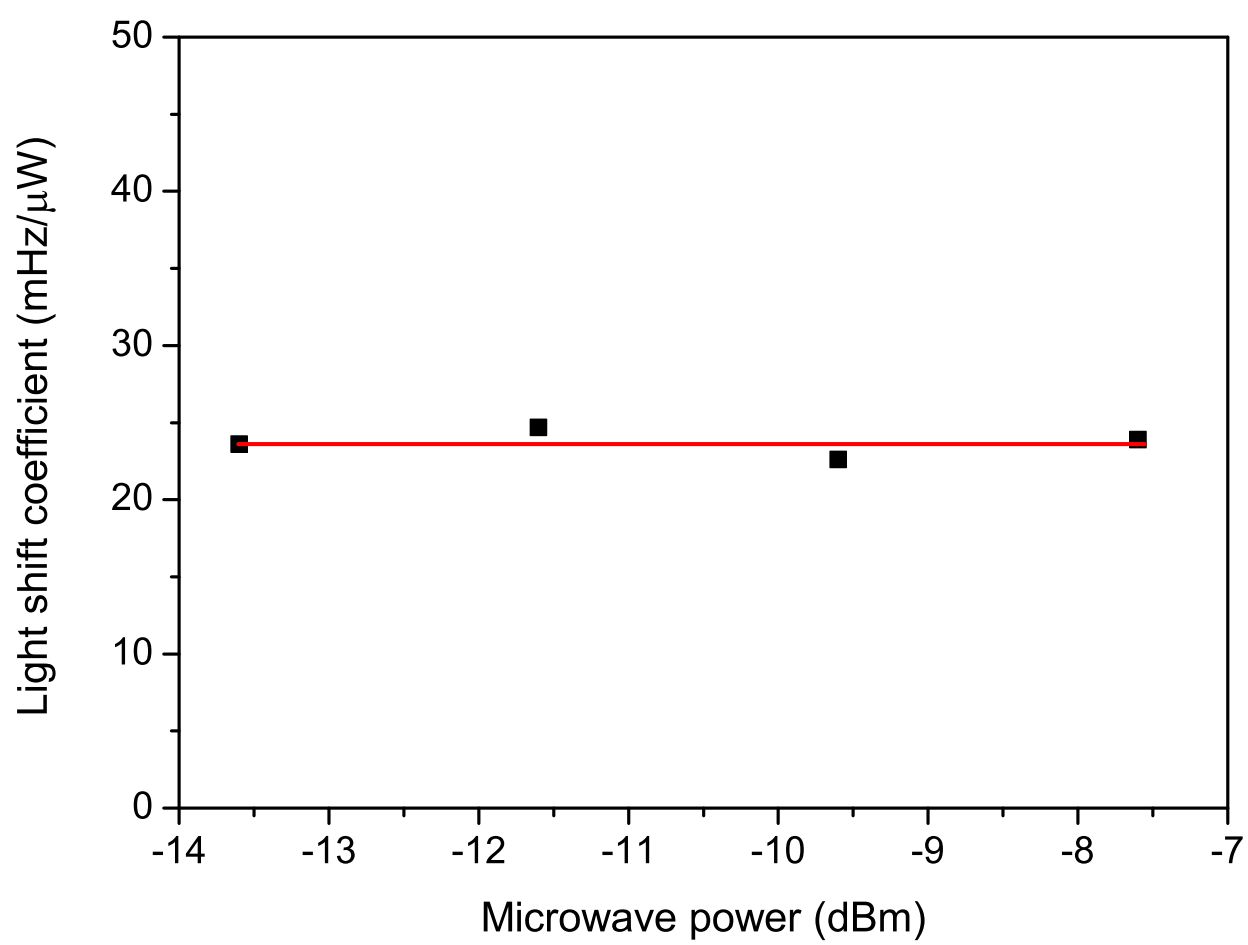

(a)

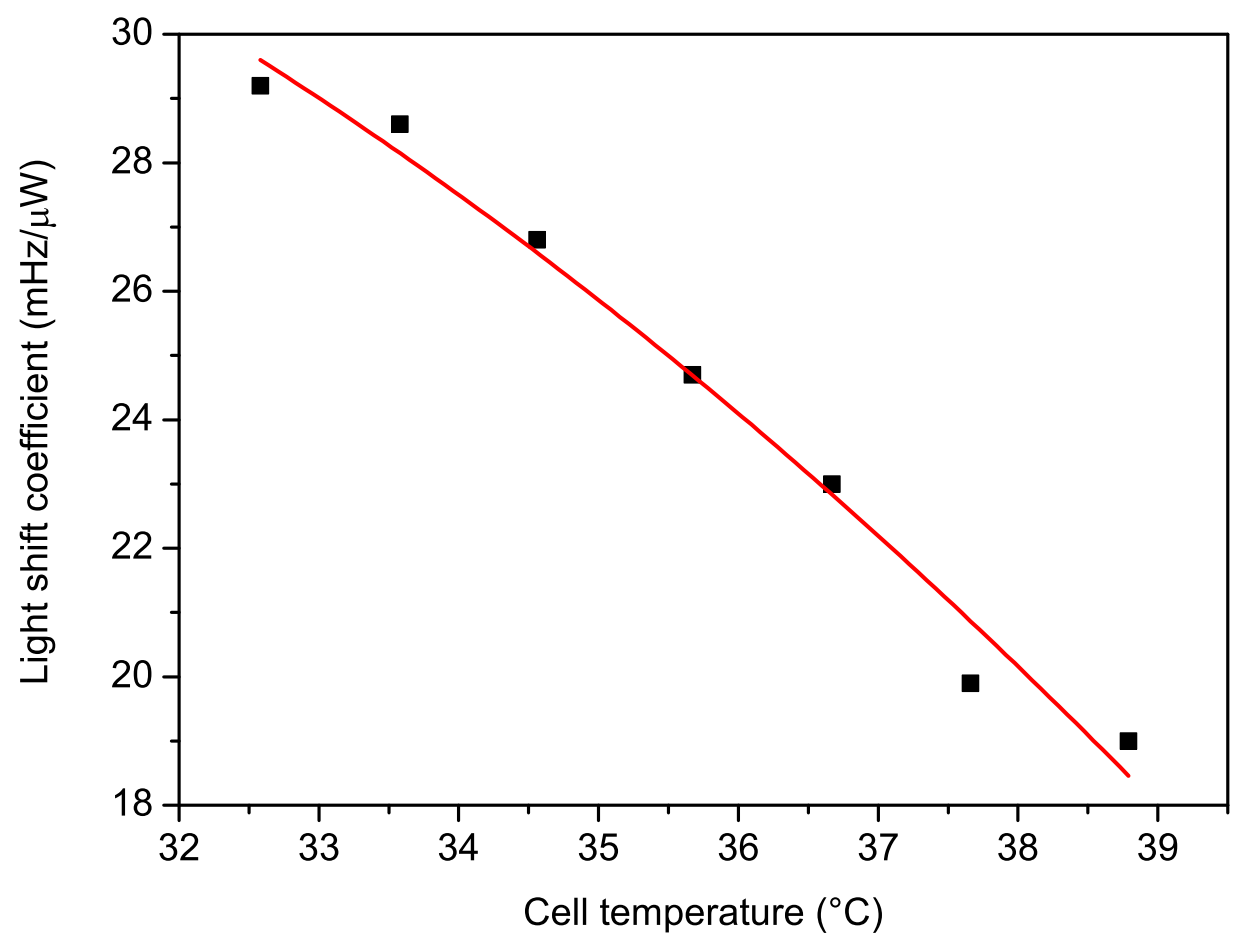

(b)

FIG. 11: 


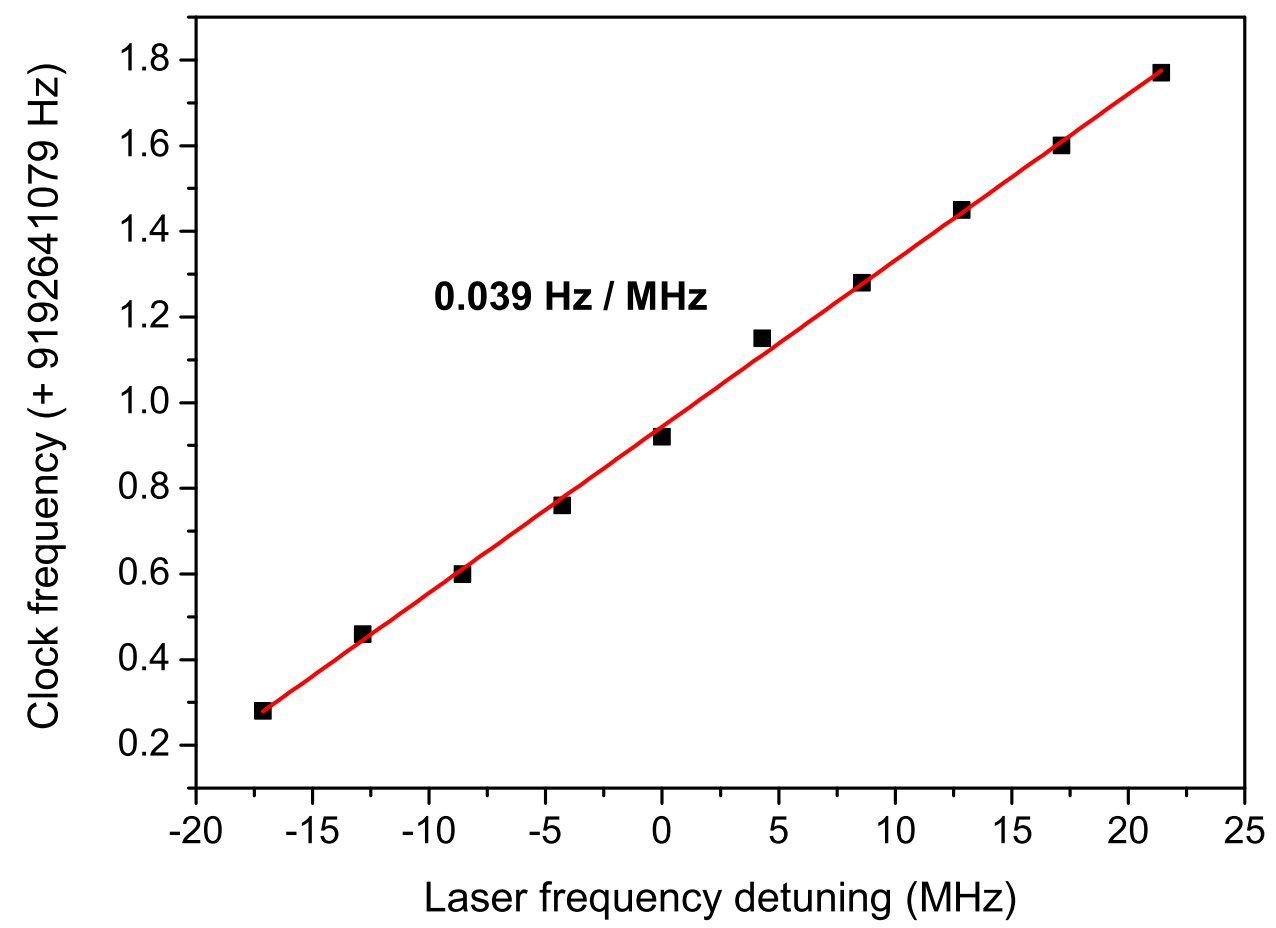

(a)

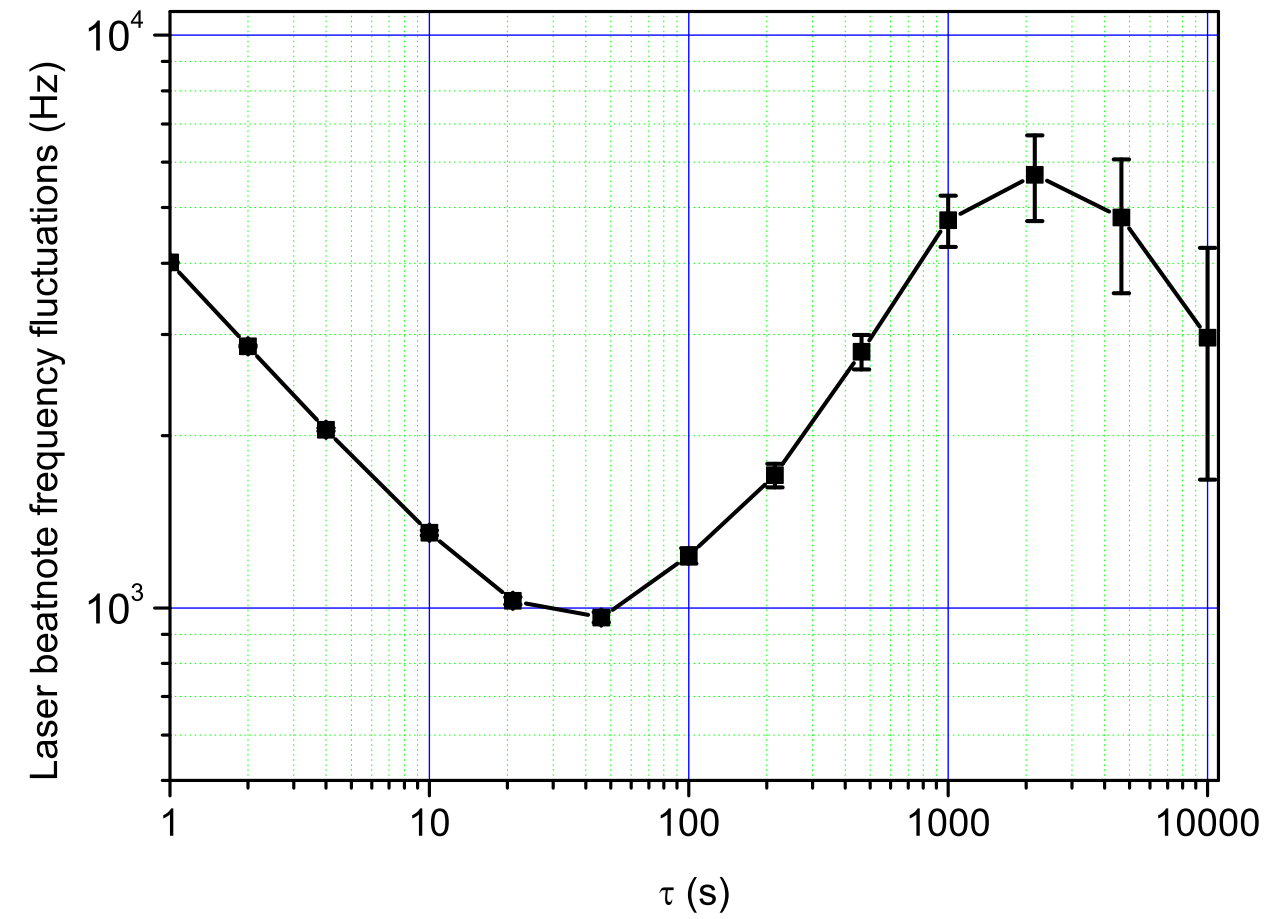

(b)

FIG. 12: 


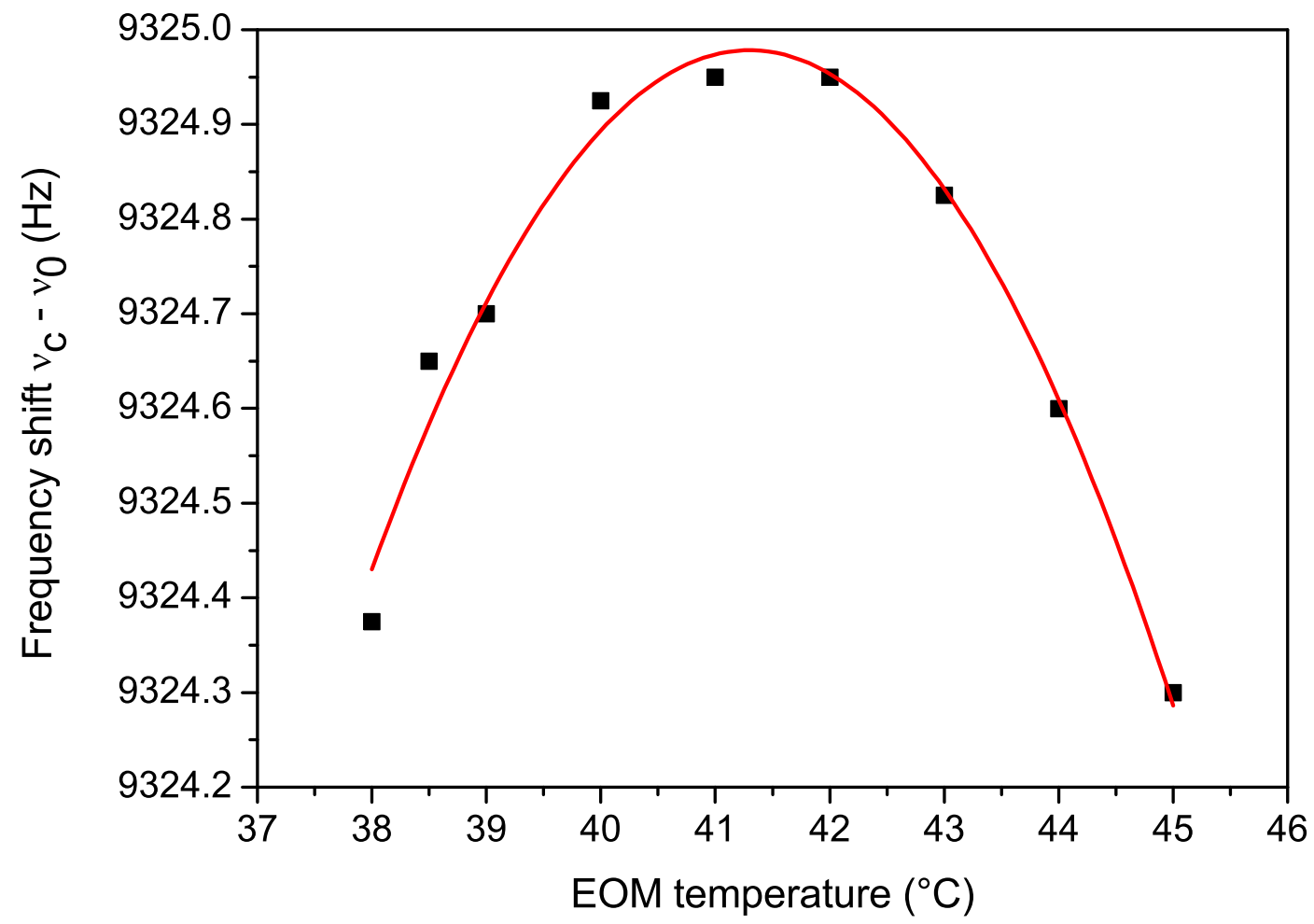

FIG. 13: 


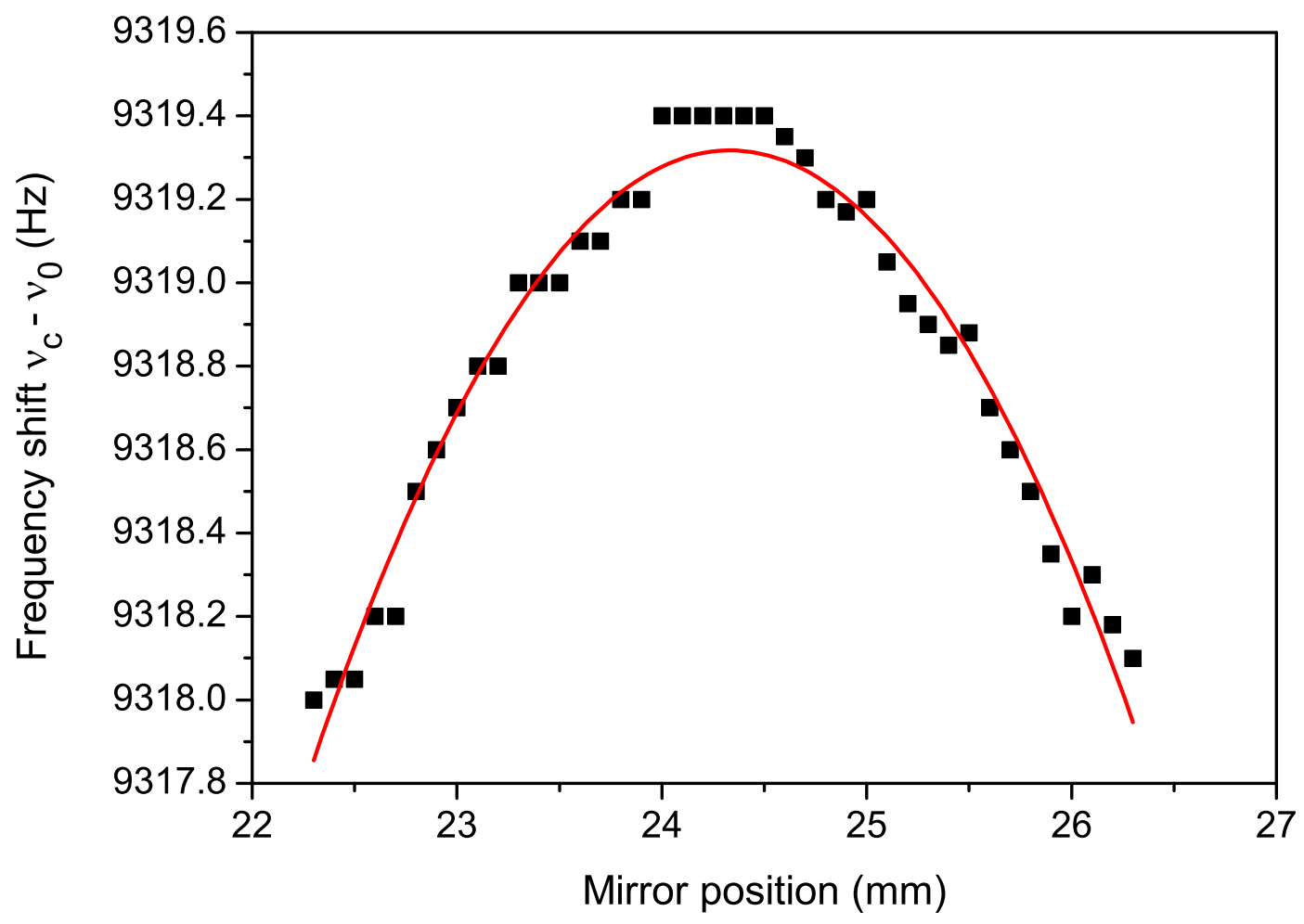

FIG. 14: 